The ecology of competition: A theory of risk-reward environments in adaptive decision making

Timothy J. Pleskac

University of Kansas, Max Planck Institute for Human Development

Larissa Conradt, Christina Leuker, \& Ralph Hertwig

Max Planck Institute for Human Development, Center for Adaptive Rationality

Author Note

All data and analyses reported in this paper can be found at this repository at the Open Science Framework: https://osf.io/jrv4y/. A draft of this paper was posted on PsyArXiv. We presented some of the formal results of the theory in 2017 at SPUDM 26. We thank Lukas Maximilian Eggeling for helping to design and administer the study on how people's subjective probabilities change with changes in the competitive environment. We thank Deborah Ain for editing the manuscript. Author contributions: Conceptualization: T.J.P., L.C., C.L., \& R.H.; Investigation: T.J.P. \& C.L.; Formal analysis: T.J.P. \& L.C.; Writing - Original Draft: T.J.P. \& L.C; Writing - Reviewing \& Editing: T.J.P., L.C., C.L. \& R.H. Correspondence concerning this article should be addressed to Timothy Pleskac (E-mail: pleskac@ku.edu). Version 3.0,07/22/2020. 


\begin{abstract}
In many choice environments, risks and rewards - or probabilities and payoffs - seem tightly coupled such that high payoffs only occur with low probabilities. An adaptive mind can exploit this association by, for instance, using a potential reward's size to infer the probability of obtaining it. However, a mind can only adapt to and exploit an environmental structure if it is ecologically reliable, that is if it is frequent and recurrent. We develop the competitive risk-reward ecology theory (CET) that establishes how the ecology of competition can make the association of high rewards with low probabilities ubiquitous. This association occurs because of what is known as the ideal free distribution (IFD) principle. The IFD states that competitors in a landscape of resource patches distribute themselves proportionally to the gross total amount of resources in the patches. CET shows how this principle implies a risk-reward structure: an inverse relationship between probabilities and payoffs. It also identifies boundary conditions for the risk-reward structure, including heterogeneity of resources, computational limits of competitors, and scarcity of resources. Finally, a set of empirical studies $(N=1,255)$ demonstrate that people's beliefs map onto properties predicted by CET, and change as a function of the environment. In sum, grounding people's inferences in CET demonstrates how the behaviors of a boundedly rational mind can be better predicted once accounts of the mind and the environment are fused.
\end{abstract}

Keywords: risk-reward structure, heuristics, uncertainty, ecological rationality, environment, ideal free distribution 
The ecology of competition: A theory of risk-reward environments in adaptive decision making

For-believe me - the secret for harvesting from existence the greatest fruitfulness and the greatest enjoyment is - to live dangerously! Build your cities on the slopes of Vesuvius! Send your ships into unchartered seas! Live at war with your peers and yourselves! Be robbers and conquerors as long as you cannot be rulers and possessors, you seekers of knowledge!

—Friedrich Nietzsche, Die Fröhliche Wissenschaft (Book 4 St. Januarius)

People have many tools they can use to navigate the inescapable uncertainties they face in the world. One such tool is probability theory. Once described as "common sense reduced to calculus" (Laplace, 1902/1814, pp. 196-197), probability theory provides a means to quantify and tabulate uncertainties by assigning probabilities to possible events, thereby turning uncertainties into measurable risks (Arrow, 1951; Keynes, 1921; Knight, 1921; Kozyreva et al., 2019; Luce \& Raiffa, 1957; Savage, 1954). Yet due to the computational limitations of the user or the limitations of the theory itself, probability theory is not a catch-all. For instance, Savage (1954) worried that probability theory and the associated subjective expected utility theory were limited to what he called small worlds, where options and events are clearly demarcated. In more ambiguous situations, he suggested using simple decision heuristics that forgo probabilities altogether.

The use of heuristics does not preclude the use of probabilities to help tame uncertainty. In fact, there are several heuristics or rules of thumb that people may use to assign probabilities to uncertain events. One common rule used by mathematicians, statisticians, scientists, and laypeople alike is the principle of indifference, whereby the possible events under consideration are treated as equally likely to occur (Keynes, 1921; Laplace, 1902/1814). Another heuristic is to use the similarity between a possible event and a large class of events as a proxy for the likelihood of it occurring (Kahneman \& 
Frederick, 2002; Tversky \& Kahneman, 1974)(see also Silver, 2012, p. 84). Here we focus on a rule for assigning probabilities that is based on a ecological relationship between probabilities and payoffs or risks and rewards, the risk-reward structure.

This structure seems to have given rise to a rule — that high rewards are unlikely — that ripples through the life lessons offered by everyone from philosophers to financial advisers. The ubiquity of this advice suggests that the risk-reward structure may be useful to a human mind seeking to handle uncertainty. Pleskac and Hertwig (2014) showed one way this structure is used is in the form of the risk-reward heuristic. To see how the heuristic works, consider a monetary gamble that costs $€ 2$ to play and offers $€ 100$ if a specific event occurs, but otherwise nothing. The risk-reward heuristic infers the unknown probability of winning a payoff $p$ from the ratio of the entry costs of playing the gamble $c$ to the total amount of possible winnings as $p=c /(c+g)$, where $g$ is the possible payoff $(€ 100)$. Thus, if the gamble costs $€ 2$ to play, the risk-reward heuristic identifies the probability of winning (assuming a fair bet) as approximately $2 \%$.

There is growing evidence that people use the risk-reward heuristic to infer missing probabilities and missing payoffs, which in turn impacts the choices they make (Hoffart et al., 2018; Pleskac \& Hertwig, 2014; Leuker et al., 2018; Skylark \& Prabhu-Naik, 2018). The risk-reward structure also impacts other aspects of decision making, including how people evaluate options with known probabilities (Leuker et al., 2019b, 2019a). Enlisting an environmental structure like the risk-reward structure-be it via a heuristic or within a larger framework, such as Bayesian inference (e.g., Chater et al., 2010; Gershman et al., 2015; Griffiths et al., 2010) or reinforcement learning (e.g., Daw et al., 2005; Niv, 2009) —is appealing because it requires relatively little time and effort; decision makers can instead invest more of their limited resources in seeking out other information or achieving other goals. The challenge is that the risk-reward heuristic (and any other cognitive process that enlists the risk-reward structure) can only operate reliably if the environmental regularity is frequent and recurrent (Barkow et al., 1995). We refer to this ecological requirement of 
an adaptive mind as the ecological reliability principle. Here we show the risk-reward structure meets this principle by developing the competitive risk-reward ecology theory (CET). CET shows that the risk-reward structure is ecologically reliable by showing that competition over limited resources, a property that itself is frequent and recurrent, provides a sufficient set of conditions for the structure to emerge. We then use CET to identify a set of boundary conditions (e.g., homogeneity of resources, computational limits of competitors, and scarcity of resources) for the risk-reward structure and show how relaxing these conditions results in systematic changes in the risk-reward structure. Finally, we examine previous datasets and report new studies to investigate the degree to which people's beliefs correspond to properties of the risk-reward structure predicted by CET. We conclude by discussing a broader point: A comprehensive theory of adaptive behavior must go beyond describing the environment to which a mind adapts; it must spell out a principled theoretical account of that environment. Only when theories environmental structures are integrated with theories of cognition can adaptive behavior be predicted.

\section{Risk-reward structure}

The risk-reward structure seems to be common in human choice environments. Take, for example, roulette. Figure 1 plots the probabilities of winning each possible payoff (relative to a $\$ 1$ bet). This risk-reward structure takes a precise form-namely, a hyperbolic function - where the chances of winning are an inverse function of the payoffs (rewards). As a result, the probability of winning decreases as payoffs increase. A survey of numerous human-made choice environments showed the same or a similar regularity in a range of environments (Pleskac \& Hertwig, 2014), including betting at the horse track, buying lottery tickets, bargaining, submitting manuscripts to scientific journals (trading acceptance rate for impact factor), and purchasing bull semen to inseminate dairy cows (trading the semen's conception rate for profitability).

In these modern environments, the risk-reward structure can typically be traced back 


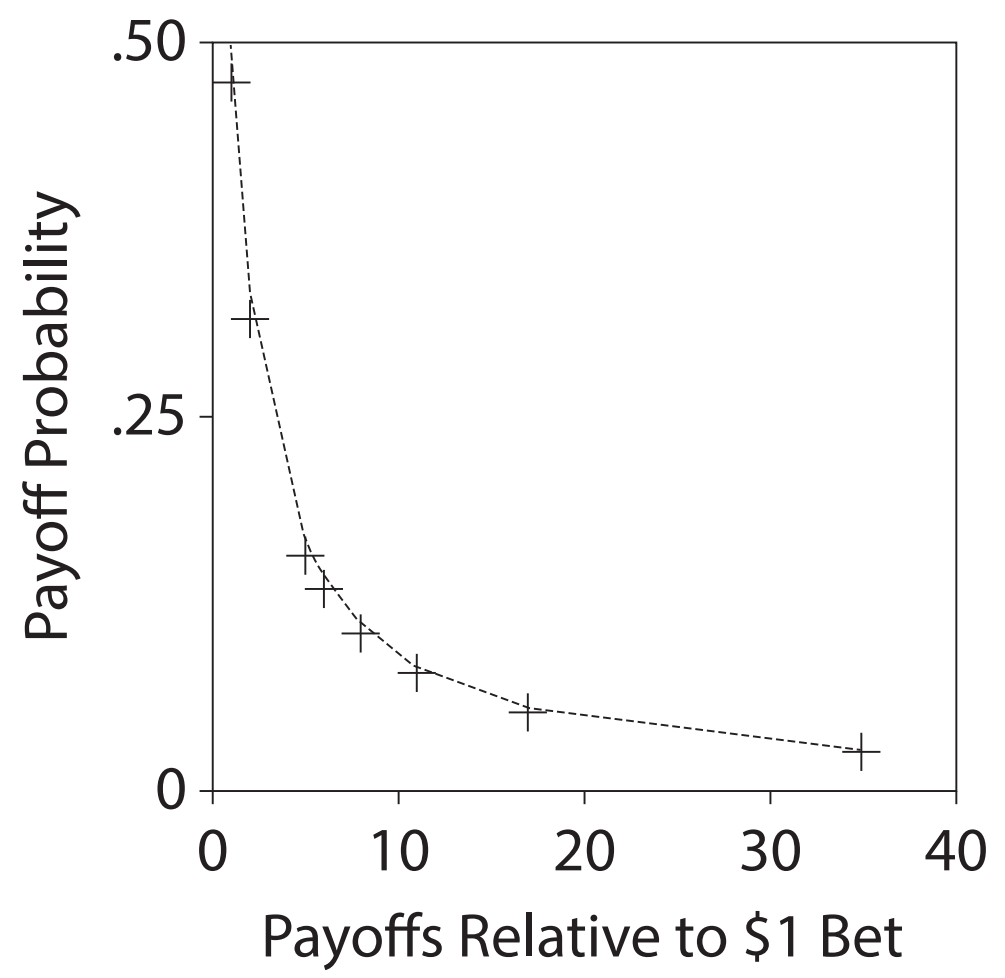

Figure 1. The risk-reward structure in roulette. The dotted line is the estimated probability assuming a fair bet. The crosses represent the actual probabilities.

to the forces of the marketplace. Buyers desire high payoffs $g$ with the highest of probabilities $p$ at the lowest $\operatorname{cost} c$. Sellers want the opposite. These opposing forces push lotteries toward a fair bet (see also Samuelson, 1965). Consequently, any increase in a potential payoff would have to be offset with an increase in cost in order to make the gamble attractive for both parties (a Pareto-efficient state). In this state, expected gains correspond to expected losses such that

$$
p \times g=(1-p) \times c .
$$

A gamble represents a fair bet if and only if the probability of winning is

$$
p=\frac{c}{c+g} \text {. }
$$

Thus, the probability of a payoff will be inversely related to its magnitude because the expected payoffs and costs of gambles are pushed toward their fair price. The dashed line 
in Figure 1 plots this fair-bet probability for roulette. In roulette, the house always wins: The actual probabilities (crosses) are systematically lower than those predicted by the assumption of fair bets (with a mean absolute deviation of .005). Nevertheless, there is a close correspondence between the actual and fair bet probabilities.

While this kind of gamble may be common, there is a much larger class of situations in which people face competition for resources, such as harvesting food, searching for a mate, or finding a new home. Even in situations such as these, which lack a clear institutionalized market with buyers and sellers, one may expect a risk-reward structure. We might attempt to investigate this hypothesis inductively, by surveying choice environments and quantifying the frequency of various risk-reward structures. This inductive form of an ecological analysis can be productive and is a common approach (e.g., Anderson \& Schooler, 1991; Brunswik, 1944; Czerlinski et al., 1999; Gibson, 1979; Hertwig et al., 2008; Şimşek \& Buckmann, 2015; Stewart et al., 2006; Todd et al., 2012; Ungemach et al., 2011; Pleskac \& Hertwig, 2014). However, its piecemeal nature makes predicting the risk-reward structure in a specific environment difficult. This limitation impacts the understanding of how adaptive choice strategies are in a given choice environment - that is, how ecologically rational they are (Marewski \& Schooler, 2011; Todd et al., 2012). In order to act in an ecologically rational manner, a person should enlist a heuristic in suitable environments and ignore it in unsuitable environments; for instance, a person should use the risk-reward heuristic only when a risk-reward structure is present. However, without a principled understanding of when and where a particular environmental structure occurs, there is no normative benchmark to which to compare a person's behavior. As a result, conclusions regarding ecological rationality are at risk of being post hoc when an ecological analysis is grounded in an inductive approach. To overcome the limitations of an inductive approaches, we instead take a deductive one identifying conditions that can give rise to a risk-reward structure. In the classes of situations we are concerned with, limited resources are distributed across patches in a resource landscape. Competitors are also distributed 
across the landscape and compete for these choice environments. We turn to this dynamic next.

\section{Limited resources and the distribution of competitors}

Competition over limited resources forms a common dynamic in many choice environments. In this situation, one critical factor in determining the probability of obtaining a resource is the number of individuals competing for it. The more competitors there are, the less likely it is that any given individual will obtain that resource. Therefore, to understand the risk-reward structure in these situations, one must first establish the distribution of competitors an individual will encounter. To do so, we draw on a well-known principle from behavioral ecology, the ideal free distribution (IFD) principle (Fretwell \& Lucas, 1969). According to this principle, competitors distribute themselves to match the total gross amount of resources within patches (see Davies et al., 2012, Chapter 5).

To get an intuition of the IFD principle, consider fishing vessels heading out to sea. Each crew wants to minimize the effort they put into catching their limit. Crews must identify the location they believe has the best chance of helping them meet this goal and compete with crews of other vessels to get there first. As they do so, a dynamic equilibrium forms so that in each fishing location there is effectively the same catch-per-unit of effort (Gillis et al., 1993; van der Lee et al., 2013). Ultimately, the number of fishing vessels in each location is proportional to the amount of fish in each location, creating an IFD of fishing vessels.

Formally, an IFD is present when the number of competitors $n_{y}$ (e.g., vessels) in each patch $y$ (e.g., fishery) is proportional to the amount of resources $r_{y}$ in each patch (e.g., the gross total amount of fish),

$$
n_{y} \propto r_{y}
$$

Many animals - including mallard ducks foraging for bread crumbs in a pond (Kennedy \& Gray, 1993), pike searching for food in lakes (Haugen et al., 2006), and bumblebees seeking 
nectar among flowers (Dreisig, 1995) — have also been shown to distribute themselves in a way that is consistent with an IFD. This relationship between amount of resources and number of competitors also extends to other types of resources, including mates (Parker, 1974), breeding sites (Alatalo et al., 1992), spawning sites (Smith et al., 2000; Valladares \& Lawton, 1991), and nesting sites (Nicolai et al., 2014). An IFD also occurs when resource patches are organized conceptually (e.g., flower types for bees) rather than spatially (Chittka et al., 1999; Waser, 1986). IFDs occur in many human situations as well; for instance, when applying for jobs (Krueger, 1988; Holzer et al., 1991) or looking for rewards in behavioral studies (Goldstone \& Ashpole, 2004; Goldstone et al., 2005; Sokolowski et al., 1999).

An IFD of competitors is a population-level phenomenon that occurs as animals or humans seek to maximize their individual resource consumption. ${ }^{1}$ The IFD principle rests on two key assumptions: first, individuals are $i d e a l$ and, second, they are free. The assumption that individuals are ideal means that individuals can detect the patches of resources that permit the highest rate of consumption. The assumption that individuals are free means that individuals can move between patches with no restrictions or costs. Both assumptions are idealized descriptions of individuals and the conditions they face. It is therefore important to both demonstrate how an IFD of competitors can imply a risk-reward structure and study how violations of the assumptions impact the predictions that follow from the IFD principle (e.g., Kennedy \& Gray, 1993; Sutherland et al., 1988; Tregenza, 1995). We now turn to the first of these two tasks: showing how an IFD of competitors can imply a risk-reward structure.

\footnotetext{
1 The IFD principle bears some resemblance to Hernstein's (1970; 1974) matching law, where an individual tends to equate the rate of a response to the rate of reinforcement. The crucial difference is that the matching law concerns behavior at the individual level, whereas the IFD principle refers to population-level behavior (see also Houston \& McNamara, 1988).
} 


\section{The IFD principle and the risk-reward structure}

To determine what the IFD principle implies about the relationship between risk and reward, we need to establish how the size of the resource $s_{y}$ (i.e., reward) and the probability of successfully obtaining the resource $p$ (i.e., risk) covary. To do so, we first break down the total amount of resources $r_{y}$ available in a given patch into the number of resources $m_{y}$, multiplied by the size of the resources,

$$
r_{y}=m_{y} \times s_{y}
$$

Thus, an IFD can be rewritten, substituting Equation 4 into Equation 3, as

$$
n_{y} \propto m_{y} \times s_{y}
$$

The probability $p_{y}$ of obtaining the resource - the success probability - is linked to both the number of resources and the number of competitors in the patch. Holding all else constant, the success probability in a patch will increase with the number of resources $m_{y}$ so that the success probability is proportional to the number of resources in patch $y, p_{y} \propto m_{y}$. The success probability will also decrease with the total number of competitors in the same patch, $p_{y} \propto 1 / n_{y}$. These two properties imply that the success probability is proportional to the ratio of the number or units of resources to competitors in a patch

$$
p_{y} \propto \frac{m_{y}}{n_{y}}
$$

The link between success probability and resource size is established by substituting Equation 5 into Equation 6. This reveals that success probability is inversely proportional to the size of the resource in a patch,

$$
p_{y} \propto \frac{1}{s_{y}} .
$$

Therefore, if there is variation in the size of resources between patches but not within patches (e.g., because different patches have different sizes of fish), then competitors like 
our fishing vessels face a choice between patches that trade resource size for success probability. That is, a basic IFD of competitors predicts that the success probability is inversely proportional to resource size across a landscape: a risk-reward structure.

Three points are worth emphasizing. First, the fact that an IFD can imply a risk-reward structure suggests that the structure is not limited to markets with institutionalized buyers and sellers, but can emerge whenever competitors vie for a finite resource distributed across a landscape. Second, by demonstrating that an IFD can imply a risk-reward structure, CET moves beyond merely describing an environment to which agents are adapted. Instead it identifies a set of conditions that can give rise to a risk-reward structure and helps establish how frequent and recurrent the structure is. Third, because the assumptions it uses can be systematically changed, CET makes it possible to examine when and how the risk-reward structure will change and even break down as a result of changes in the ecology. We turn to this topic next.

\section{The Risk-Reward Structure and Changes in the Ecology of Competition}

In order to diagnose and predict adaptive behavior one must first establish the conditions under which the ecological structure is fully present, weakly present, or absent altogether. To this end, CET reveals at least eight ecological conditions that can directly implact the risk-reward structure (Table 1). Two conditions are relatively straightforward. The first relates to equilibrium: An IFD of competitors is expected to be present when the average consumption of resources is equal across patches. If the system cannot, or has yet to reach this equilibrium, the distribution of competitors should not conform to an IFD and a reliable inverse relationship between the success probability and resource size is not expected (see Table 1, assumption 1). This condition implies, for instance, that a newly forming ecology (e.g., a new market) will initially not have a risk-reward structure.

The second condition pertains to the availability of resources. An IFD of competitors is only expected when resources are limited. When resources are unlimited (such as in 
some cases air), agents do not have to compete with each other and maximize their consumption of resources. Consequently, no IFD develops. This implies that a risk-reward structure should not be expected for unlimited resources, where the success probability will be essentially one for all resource sizes (see Table 1, Assumption 2). Both of these conditions - equilibrium and limited availability of resources - thus establish boundary conditions for the emergence of a risk-reward structure. We now turn to analyze the remaining six boundary conditions and quantify how they systematically shape the risk-reward structure. 
Table 1

Boundary Conditions of the Risk-Reward Structure in Ecologies of Competition Over Limited Resources

\begin{tabular}{|c|c|c|}
\hline Ideal Free Distribution (IFD) Assumption & Violation of the Assumption & Does the Risk-Reward Structure Still Hold? \\
\hline (1) System is in equilibrium. & Rate of resource consumption is not equal across patches. & $\begin{array}{l}\text { When the system is not in equilibrium, an IFD does not hold and no reliable risk-reward } \\
\text { structure is expected. }\end{array}$ \\
\hline (2) Limited availability of resources. & Resources are unlimited. & $\begin{array}{l}\text { When resources are unlimited an IFD does not hold and no risk-reward structure is ex- } \\
\text { pected. }\end{array}$ \\
\hline $\begin{array}{l}\text { (3) Homogeneous resource sizes within } \\
\text { patches. }\end{array}$ & Heterogeneity in resource size within patches. & $\begin{array}{l}\text { As within-patch variability in resource size increases, the success probability equalizes across } \\
\text { all sizes, reducing the risk-reward structure. }\end{array}$ \\
\hline $\begin{array}{l}\text { (4) Landscape saturation of competitors } \\
\text { and/or resources. }\end{array}$ & $\begin{array}{l}\text { Competitors and/or resources are sparsely distributed across } \\
\text { the landscape. }\end{array}$ & $\begin{array}{l}\text { As landscape saturation decreases, the risk-reward structure weakens and becomes more } \\
\text { disperse at low resource sizes. }\end{array}$ \\
\hline (5) Competitors are ideal. & $\begin{array}{l}\text { Competitors' ability to discriminate between the quality of } \\
\text { the patches is limited. }\end{array}$ & $\begin{array}{l}\text { As the ability to discriminate decreases, the risk-reward structure weakens and becomes } \\
\text { more disperse at low resource sizes. }\end{array}$ \\
\hline (6) Competitors are equally competitive. & Competitors have different competitive abilities. & The risk-reward structure holds within categories of abilities, but not across categories. \\
\hline $\begin{array}{l}\text { (7) Competitors do not interfere with } \\
\text { each other in the acquisition of resources. }\end{array}$ & Interference is possible in obtaining resources. & $\begin{array}{l}\text { The risk-reward structure holds, but the distribution of competitors will be inelastic (less } \\
\text { than proportional) with respect to resource size. }\end{array}$ \\
\hline $\begin{array}{l}\text { (8) Competitors only require a single re- } \\
\text { source type. }\end{array}$ & Competitors require multiple different limited resources. & $\begin{array}{l}\text { The more limiting the resource, the more reliably the risk-reward structure can be expected } \\
\text { to emerge. }\end{array}$ \\
\hline
\end{tabular}




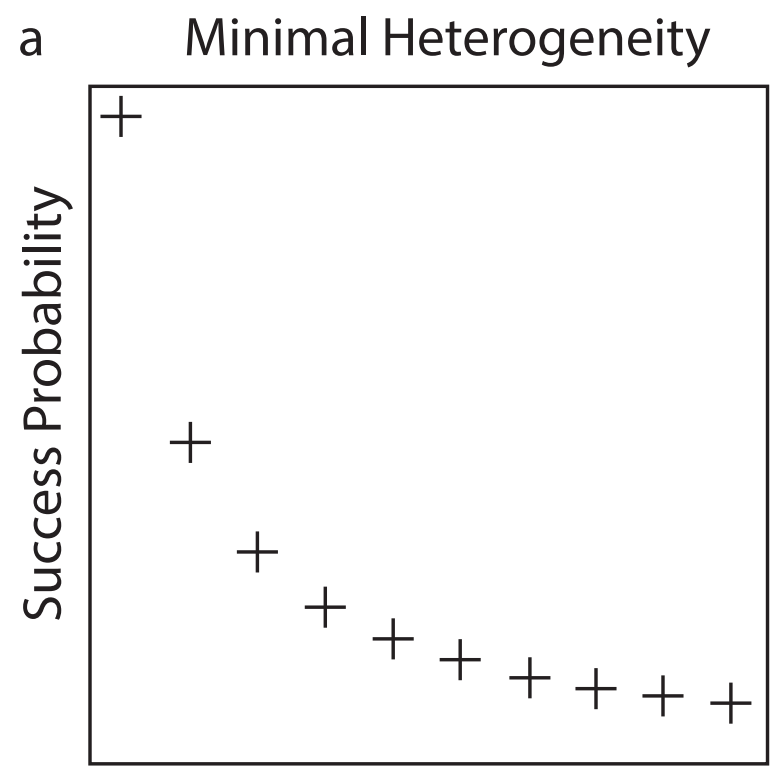

b

Low Heterogeneity
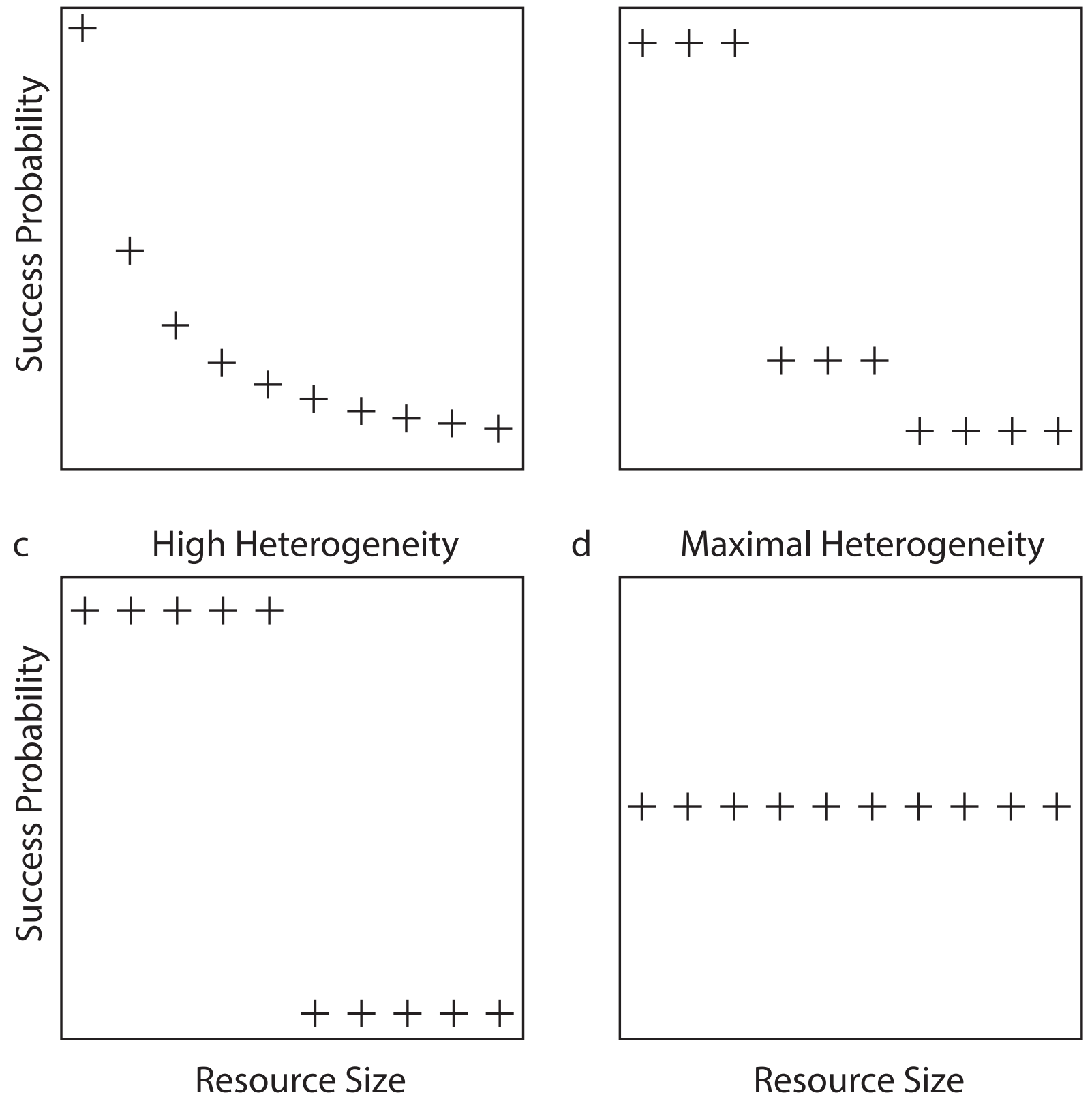

Figure 2. Success probability as a function of resource size in landscapes with different levels of resource size heterogeneity within patches. Within-patch heterogeneity of resource size increases from panels a to d. For details on how the probabilities were estimated see Appendix A. 


\section{Heterogeneity in resource sizes}

Although the assumption that resource sizes are approximately equivalent within patches is a plausible approximation in some cases (e.g., apples in an orchard), there are also situations where resource sizes vary within patches. Indeed, Appendix A proves that for landscapes made up of two resource sizes (large and small) the risk-reward structure degrades when the patches have more heterogeneous resource sizes. With maximal heterogeneity of resource sizes, there will be no risk-reward structure.

We simulated how heterogeneity in resource size impacts the risk-reward structure using more than two resource sizes (Figure 2; for details see Appendix A). At minimal within-patch heterogeneity of resource sizes, the success probability is inversely related to resource size (Figure 2, panel a). At maximal heterogeneity, the success probability is identical for all resource sizes (panel d). For intermediate levels of heterogeneity, the risk-reward structure falls within these two extremes (panels b and c). Notably, in all of these cases, even as within-patch heterogeneity increases, an IFD is still present. Thus, when there is competition over finite resources, an IFD can be present without a risk-reward structure.

\section{Lack of landscape saturation}

Another assumption of the IFD principle is that the landscape is sufficiently saturated in terms of both resources and competitors. If the number of competitors or the total number of resources is relatively small compared to the number of patches, some patches may attract relatively few or even no competitors. This change in the saturation of the landscape - in terms of the number of resources or the number of competitors - impacts success probabilities. To find out how saturation impacts the risk-reward structure, we simulated four landscapes, each with different levels of saturation of competitors and/or resources (Figure 3). In general, the risk-reward structure is robust. There are some quantitative changes to the structure: A comparison of the top two panels in Figure 3 (low 
Low Competitor,

a Low Resource Saturation

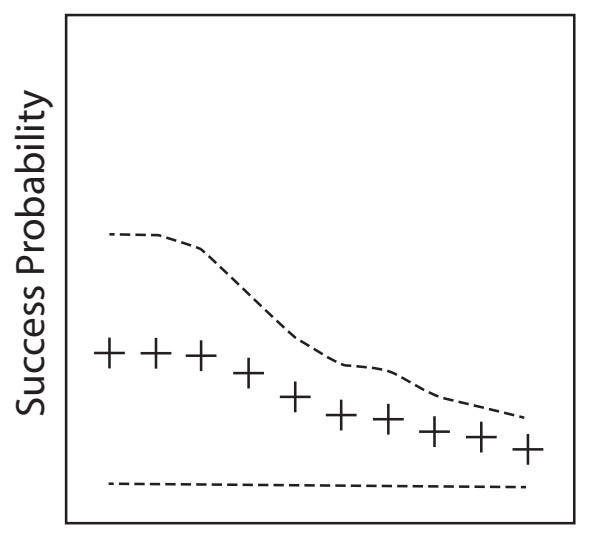

High Competitor,
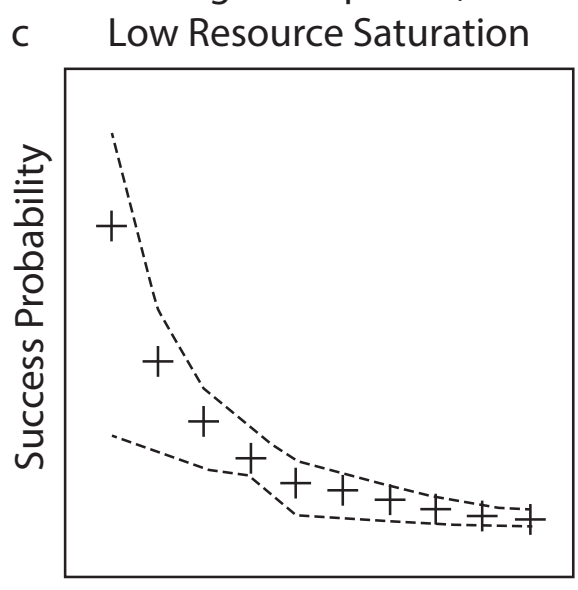

Resource Size
Low Competitor,

b High Resource Saturation

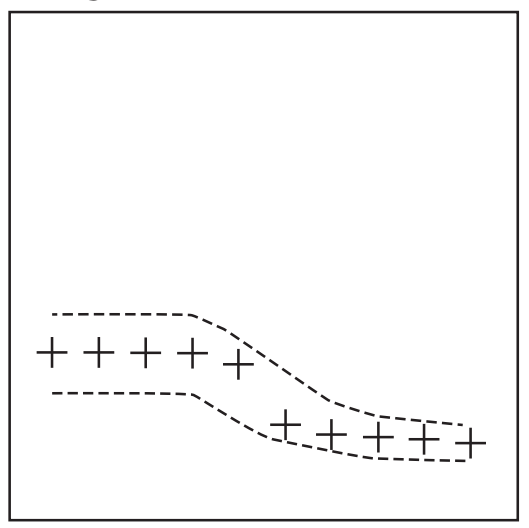

High Competitor,

d High Resource Saturation

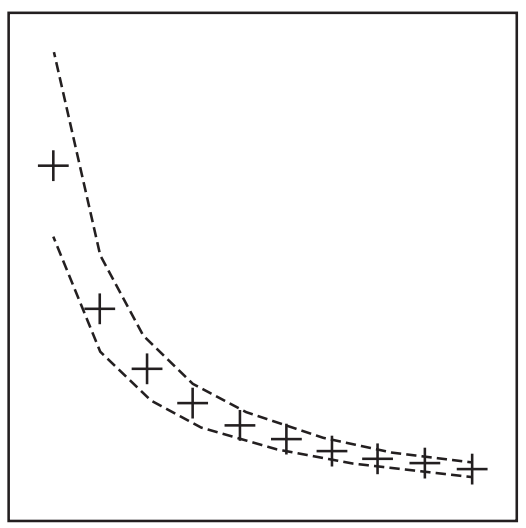

Resource Size

Figure 3. Success probability as a function of resource size in an IFD model with different levels of landscape saturation. The median success probability $(+)$ decreases with resource size. The fewer competitors there are within the landscape, the shallower the decrease. The dashed lines plot the $2.5 \%$ and $97.5 \%$ quantiles of success probabilities, showing that the fewer competitors and resources in the landscape, the greater the variability in success probabilities - particularly at smaller resource size. In each simulation, there were 200 patches with either 100 (low) or 1,000 (high) competitors and either 100 (low) or 1,000 (high) resources per size. The results are based on 2,000 simulations per parameter combination. See Appendix B for further details. 
competitor saturation in the landscape: 100 total competitors across 200 patches) to the bottom two panels (high competitor saturation: 1,000 total competitors across 200 patches) shows that fewer competitors (and thus lower saturation) result in a shallower slope. Moreover, the variation in the success probability for small resource sizes is higher when either the total number of competitors is small or resources are sparse. Thus, in less saturated landscapes, there is no guarantee that small resources will always be associated with a high success probability.

In landscapes with low resource saturation, this heteroscedasticity in success probabilities - where their variability decreases as resource size increases - has implications for how reliably resource size can predict success probabilities. With smaller resources, large variability means that the size of the resource is an unreliable predictor of success probability. However, our simulations show that this problem does not arise to any noteworthy degree with large resources: Even in sparse landscapes, the variability in success probability is low for large resources (Figure 3). For instance, in the simulation with low competitor saturation and low resource saturation the range of success probabilities for the smallest resources is 3.6 times larger than for the largest resources. As we see next, a similar regularity with regard to the heteroscedasticity of success probabilities emerges when competitors' cognitive constraints are taken into account.

\section{Nonideal individuals}

Perhaps the boldest assumption of the IFD principle is that competitors are ideal - that is, they are sensitive to the patches' rate of consumption and move to those that maximize their own success rate. Like the equally optimized homo economicus, the ideal individual of the IFD principle boasts a complete representation of the environment and unlimited cognitive resources (see Gigerenzer \& Selten, 2002; Simon, 1955). What are the consequences of a more realistic portrayal of competitors? Abrahams (1986) demonstrated that if competitors have a limited ability to detect differences in the quality 
of a patch, they will overuse poor patches and underuse rich patches. This pattern suggests a reformulation of the IFD principle from Equation 3 to

$$
n_{y} \propto r_{y}^{\alpha}
$$

The parameter $\alpha(0<\alpha<1)$ captures the degree to which competitors, in the aggregate, mismatch with respect to the resource amount. As sensitivity to patch quality increases, the distribution of competitors increasingly conforms to an IFD, reflected in an increase in $\alpha$. Kennedy and Gray (1993) found that across 52 animal studies, an estimate of $\alpha=0.66$ gave the best account of the data.

Diminished sensitivity to the quality of the patches changes the distribution of competitors, thereby impacting the risk-reward structure. To see how, recall that the amount of resources in a patch is $r_{y}=m_{y} \times s_{y}$ (Equation 4). As a result, the predicted distribution of competitors will be

$$
n_{y} \propto\left(m_{y} \times s_{y}\right)^{\alpha}
$$

Using this more general version of the IFD principle, we can determine how nonideal individuals will impact the success probability. This is done by incorporating Equation 9 into the assumption where $p_{y} \propto m_{y} / n_{y}$ (Equation 6). Doing so shows that the success probability with nonideal individuals is

$$
p_{y} \propto \frac{m_{y}^{1-\alpha}}{s_{y}^{\alpha}} .
$$

As Equation 10 implies, once the limited sensitivity of competitors is acknowledged, success probability $p_{y}$ is proportional to both the size of the resource $s_{y}$ and the number of resources $m_{y}$. If there is no variability in the number of resources across patches, then $m_{y}$ will be a constant and thus will be factored out of the equation due to proportionality. Consequently, in landscapes where each patch has the same number of resources but competitors are nonideal, success probability is less than inversely proportional to resource 


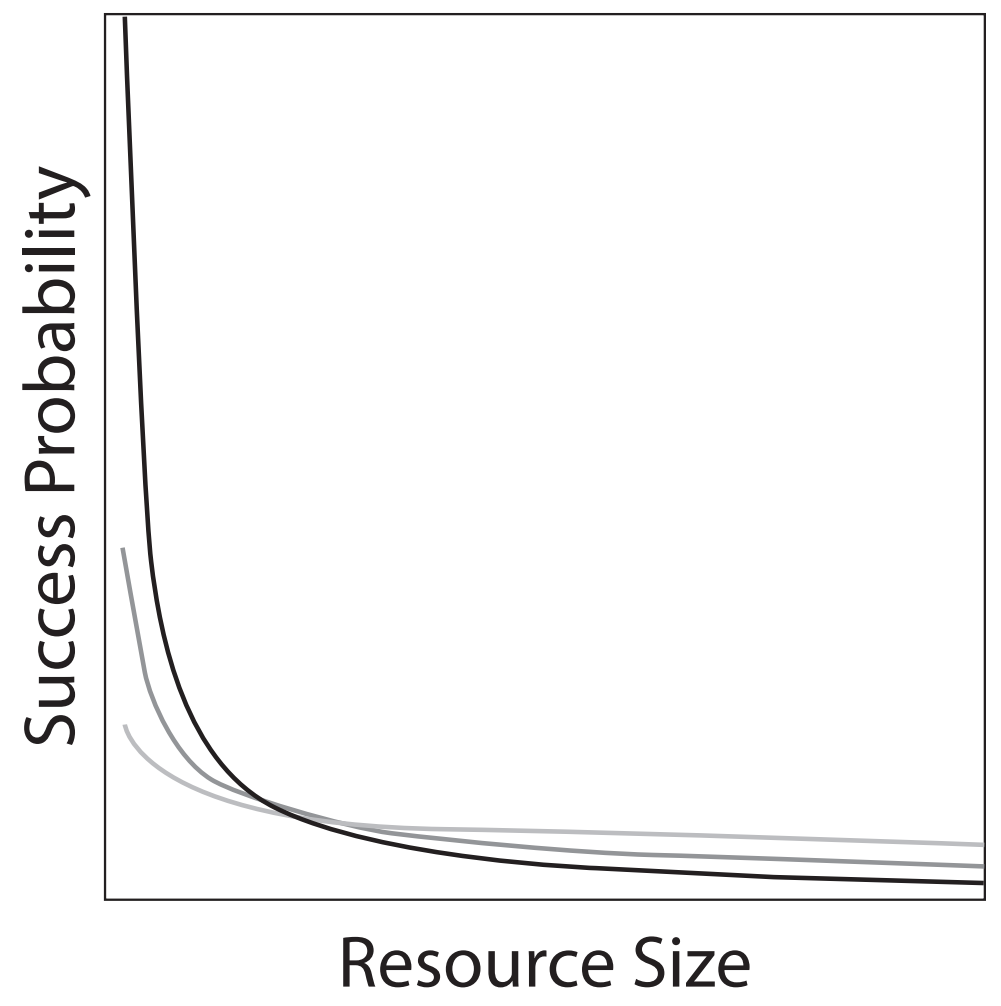

Figure 4. Success probability as function of resource size for different levels of sensitivity to patch quality $\alpha(\alpha=.3, .6$, and .8). As sensitivity to patch quality lessens and competitors become less ideal (indicated by lighter lines), success probabilities become less extreme for small resources and more extreme for large resources.

size, $p_{y} \propto 1 / s_{y}^{\alpha}$. This means that a landscape with nonideal competitors produces a flatter risk-reward structure than does one with ideal competitors (Figure 4).

We can also examine what happens if the number of resources varies between patches. If the median number of items per patch is similar for patches with different resource sizes, the median success probability still decreases with resource size $s_{y}$, just as before, but it will be shallower than what is predicted for a landscape with ideal individuals (Equation 10). However, because there is variability in the number of resources there will be variability in the success probability for a given resource size. To see the impact of the variability, assume, for example, that the $95 \%$ range of number of resource items per patch spans from 
a
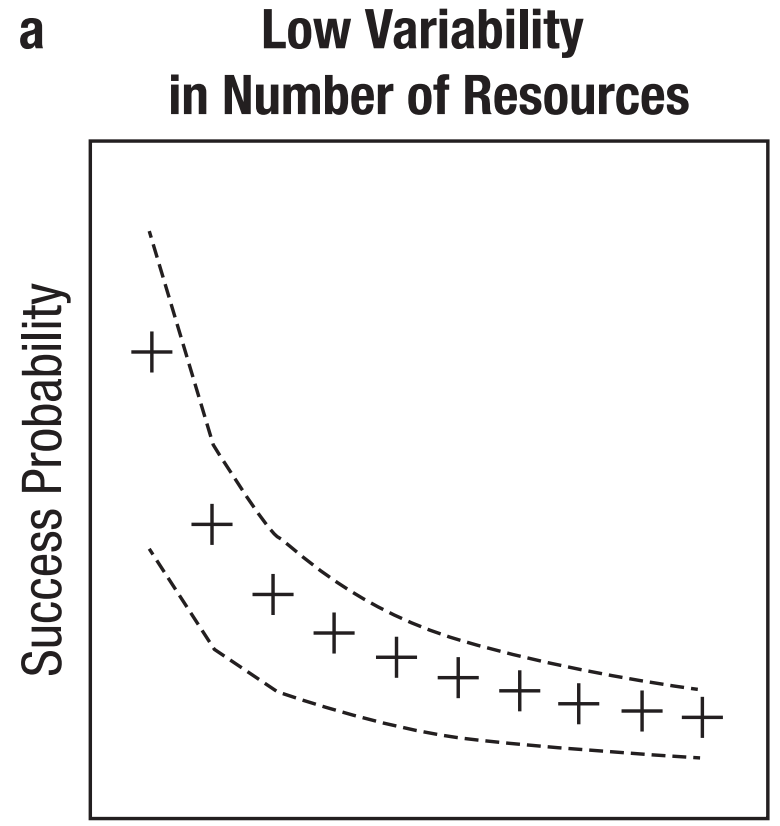

Resource Size b High Variability in Number of Resources

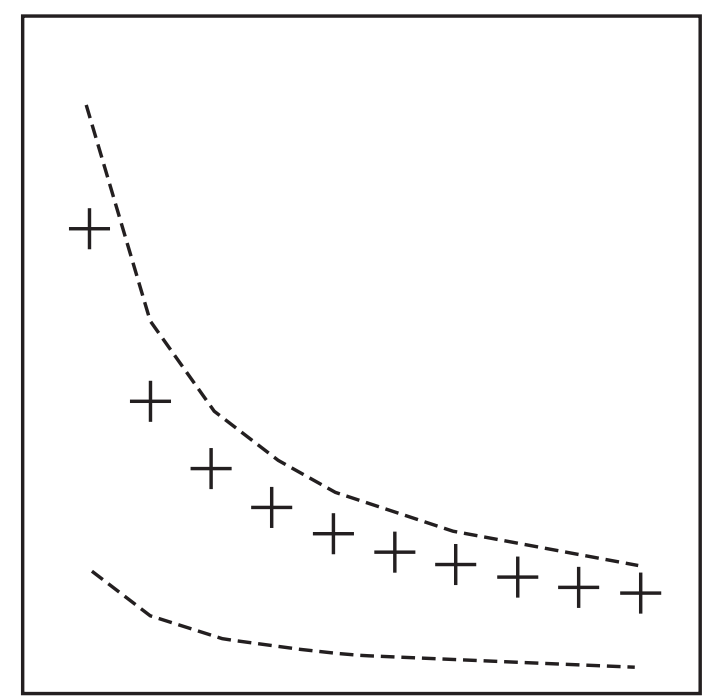

Resource Size

Figure 5. Success probability as function of resource size when constraints lead to competitors undermatching patch resources. The median success probability $(+)$ decreases with resource size. The $95 \%$ ranges of success probabilities at each resource size (dashed line) depends on the variation in the number of resources per patch (left panel: $\dot{m}=100$ to $\ddot{m}=1000$; right panel: $\dot{m}=10$ to $\ddot{m}=1000$ ).

$\dot{m}$ to $\ddot{m}$. In landscapes with nonideal individuals, Equation 10 implies that the $95 \%$ range of the predicted success probabilities will span from values proportional to $\dot{m}^{1-\alpha} / s_{y}^{\alpha}$ to $\ddot{m}^{1-\alpha} / s_{y}^{\alpha}$. Figure 5 shows how nonideal individuals in these landscapes change the range in possible success probabilities as a function of resource size. The pattern is similar to that of sparse landscapes (Figure 3). The success probability associated with a small reward item varies widely; in many of these cases the success probability will be low. In contrast, the success probability for a large resource item is less variable and nearly always low.

In sum, a limited ability to discriminate between patches of different qualities results in a risk-reward structure that is less extreme for small resources and more extreme for large resources compared to a risk-reward structure that occurs in landscapes with an IFD. 
Furthermore, if there is variability in resource sizes across patches, the presence of nonideal competitors results in an asymmetry in the relationship between risks and rewards. Much like the pattern of heteroscedasticity of success probabilities in landscapes with low resource and competitor saturation, small resource sizes preclude reliable predictions of success probabilities, while large resources are reliably linked to low success probabilities.

\section{Unequal abilities between competitors}

The IFD principle assumes that all individuals are equal in their ability to obtain a resource. This is a gross simplification; in reality, competitors often differ (Houston \& McNamara, 1988; Parker \& Sutherland, 1986; Sutherland et al., 1988). We show in Appendix $\mathrm{C}$ that if individuals differ in their competitive ability, an IFD of competitors results in a risk-reward structure within each class of competitors with the same abilities. However, the risk-reward structure is not present between classes. Instead, competitors with greater competitive ability will have greater success probabilities, regardless of resource size. A corollary of this result is that the risk-reward structure will appear weaker in these environments when ignoring, or averaging across, abilities or classes.

\section{Interference between competitors}

The IFD principle assumes that competitors do not interfere with each other's acquisition of a resource. In the real world, however, they often do just that (Sutherland, 1983), and not always because of competition (e.g., predators may frighten off prey). Interference between competitors creates a landscape where the distribution of competitors is less than proportional to the size of the rewards (Parker \& Sutherland, 1986). Despite this change in how competitors distribute themselves across resource patches, interference

does not alter the risk-reward structure (for a formal proof see Appendix D). The intuition behind the result is that interference cancels itself out by impacting both the distribution of competitors in a patch and the success probability of any given competitor. The risk-reward structure is therefore retained. 


\section{Multiple resource types}

Finally, what happens to the risk-reward structure when competitors in a landscape require more than one type of resource (e.g., water, food, mates)? Multiple resource types may render it impossible to achieve equilibrium across all types of resources simultaneously. For example, if most of the food is located in specific patches and most of the mating opportunities are in other patches, then the distribution of competitors between those patches can reflect the goal of consuming food, the goal of finding mates, or some compromise thereof. How does this affect the risk-reward structure? A possible answer comes from applying what is known in behavioral ecology as Liebig's law (Bloom et al., 1985; Danger et al., 2008; Farrior et al., 2013; Gorban et al., 2011; Harpole et al., 2011; Rosenheim et al., 2010). According to Liebig's law, growth is not determined by the total amount of resources, but by the most limiting (i.e., essential and scarce) one. The more limiting a resource type is, the more likely it is that its distribution dictates the distribution of competitors. For example, in a drought, water might be the most limiting resource, whereas in a wet season, mates or food might be more limiting. In general, however, the more limiting a resource is, the stronger the risk-reward structure is for that resource.

In summary, CET establishes how competition over limited resources results in the emergence of a risk-reward structure. CET builds on the IFD principle from behavioral ecology and establishes that an inverse relationship between the size of a resource and the probability of obtaining it is to be expected whenever there is competition over finite resources. It also establishes how robust the risk-reward structure is against changes in the ecology of competition, showing how changes in resources across the landscape and in competitors systematically affect the risk-reward structure. This structure proves to be relatively robust: The strength of the relationship weakened in various ecologies, but never reversed. If the risk-reward structure is weakened, the success probabilities of smaller rewards were often more affected than those of larger rewards. 


\section{Do people's probability estimates map onto the ecological properties of the risk-reward structure?}

CET can play an important role in understanding adaptive cognition: Given that competition over finite resources is frequent and recurrent, CET establishes that the risk-reward structure can also be expected to be frequent and recurrent. Thus, the CET shows that the risk-reward structure can meet the ecological reliability principle for an adaptive mind to make use of the risk-reward structure. This, in turn, leads to the prediction that people's estimated probabilities of different payoffs track the ecological probabilities and their link to potential payoffs in competitive ecologies. We tested this prediction in two ways. First, we examined several datasets from a lottery task to investigate whether people's representations reveal signatures of risk-reward structures that emerge from competition over limited resources. Second, in a new study, we directly manipulated the ecological conditions and asked participants to estimate the probability of successfully obtaining a resource.

Before continuing, it is important to clarify two issues regarding the empirical question of whether people's probability estimates map onto the ecological properties predicted by CET. First, CET is a theory about the environment and the relationship between resource sizes and the probability of obtaining those resources (i.e., success probabilities). It is not a theory about people's cognition. However, an important premise behind any adaptive approach to cognition (i.e., one that analyzes the world to understand the mind and how it adapts to its surroundings) is that there is some correspondence between cognition (e.g., beliefs) and environment (e.g., risk-reward structure). Proponents of an adaptive approach to cognition have conceived of this correspondence in different terms. Shepard (2001) viewed the mind as a mirror, reflecting long-standing physical aspects of the world. However, given the myriad of cognitve and perceptual limitations, a direct reflection of the environment seems implausible. Simon (1990) advanced a different view, suggesting that the correspondence between mind and environment was more akin to 
a pair of scissors: The mind and world must fit together like blades of scissors in order to produce successful behavior. Accordingly, cognition need not mirror the environment, but the two must be closely synchronised in order for cognition to function adaptively. This view implies that establishing the degree to which people's estimates converge with or diverge from structures of the environment does not necessarily strengthen or weaken the ecological theory itself. Yet, convergence or divergence do speak to how useful the theory is for understanding and predicting people's cognition and, more generally, the adaptive mind.

The second issue is that CET is a theory about a population-level phenomenon across a landscape made up of resource patches. Thus, the predictions derived from the theory are about the link between probabilities and resource sizes in a patch and across patches. This characteristic, in turn, shapes the predictions one might make about people's representations of the risk-reward relationship. Consider the heteroscedasticity property, where the variability of success probabilities decreases with increasing payoffs. This environmental property does not necessarily imply that people's internal representations show more variability at low levels of payoffs. Rather, if one assumes that people experience different patches, there might be more variability in the estimated probabilities at low payoffs across individuals.

\section{Do people's estimates reflect the ecological probabilities expected from a risk-reward structure?}

Introduction and methods. As a first step, we examined data from tasks first used by Pleskac and Hertwig (2014). There are subtle variations within the task, but all variants asked participants to consider a bet like the following:

Imagine you have been asked to play the following lottery. The lottery offers the opportunity to win $€ x$, but it costs you $€ 2$ to play. If you choose to play you would pay the $€ 2$ and, without looking, draw a ball from a basket. In the basket there are 1,000 balls. The balls are either black or red. If the ball is red 
you will win $€ x$; otherwise, if the ball is black you will receive nothing. Thus, the number of red balls in the basket determines the probability that you will win. You are less likely to win the $€ 2$ the lower the number of red balls in the basket.

Different participants saw different total payoffs $x$ (e.g., $€ 2.5,4,10,20$ ), and were asked two questions: (1) How many red balls do you think are in the basket? and (2) Would you pay $€ 2$ to play?

Here we focus on participants' responses to the first question and ask to what extent their estimated probabilities (via the estimated number of red balls in the basket) reveal two properties that CET predicts to occur frequently. The first property pertains to the relationship between risks and rewards at the extreme levels of rewards. Recall that when resource sizes are heterogeneous and when competitors have diminished sensitivity to patch quality, the risk-reward structure degrades, making success probabilities less extreme for small resources and more extreme for larger resources. In other words, the distribution becomes flatter across the possible rewards. According to this property, participants' estimates should be flatter relative to a perfect inverse relationship between payoffs and probabilities. The second property pertains to the variability in the probabilities. As CET suggests, when landscapes are lacking in terms of resources or competitors, or when competitors are not perfectly discriminating between resource sizes, the variability in success probabilities across patches decreases as resources increase; that is, there is systematic heteroscedasticity in the success probabilities. This property implies a similar relationship in participants' estimates.

We drew on probability estimates across three studies to examine whether people's responses contain signatures of (i) a flatter risk-reward structure and (ii) heteroscedasticity in the probabilities as a function of resource or reward size. The first two studies are reported in Pleskac and Hertwig (2014). In the first study, which took place in a behavioral lab, participants $(N=138)$ could win and lose real money by betting on an urn containing 
Table 2

Interquartile Range (IQR) of the Probability Estimates at Different Payoff Levels

\begin{tabular}{cc}
\hline Payoff & IQR (estimates) \\
\hline 1.25 & .30 \\
2 & .35 \\
5 & .20 \\
10 & .30 \\
25 & .29 \\
50 & .19 \\
100 & .10 \\
250 & .10 \\
500 & .10 \\
5,000 & .02 \\
\hline
\end{tabular}

100 balls. In the second study, which took place online, participants $(N=196)$ answered questions about a hypothetical bet on an urn containing 1,000 balls. The third study was a new online study in which participants $(N=455)$ also answered questions about a hypothetical bet on an urn with 1,000 balls (see Supplementary Materials for full details of the third study).

Results. Figure 6 plots the estimated probabilities of success (via the number of red balls participants estimated to be in the basket) across three studies $(N=789)$. Participants' estimates (dots, light gray) reflect an inverse risk-reward structure. Consistent with the first property predicted by CET, estimates were flatter than predicted by a perfect inverse risk-reward structure (dashed line). Moreover, consistent with the second predicted property of heteroscedastity, the estimates also became less variable as the reward size increased (see Figure 6 and interquartile ranges of probability estimates in Table 2). 


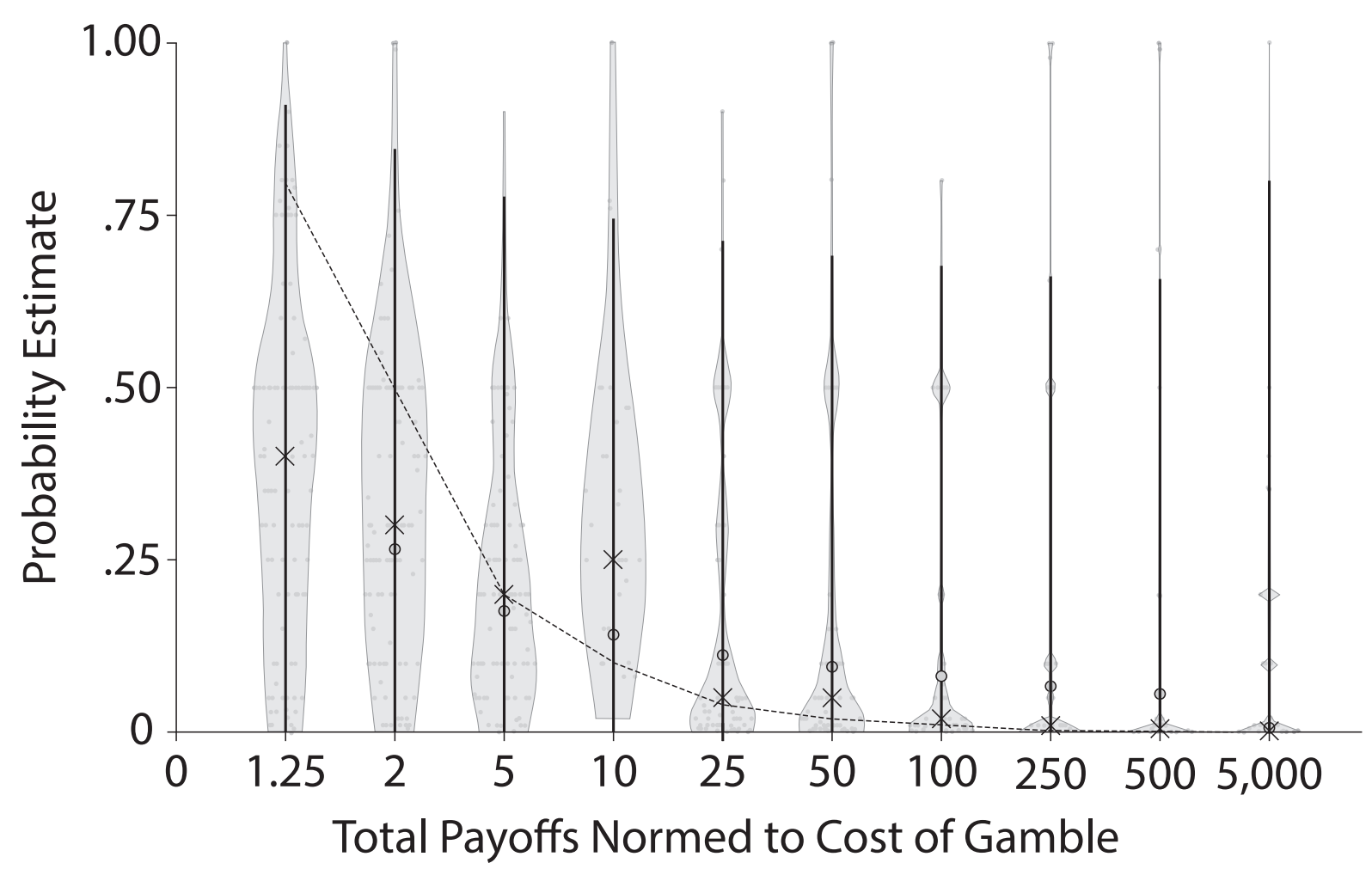

Figure 6 . Probability estimates by reward magnitudes from the lottery task. The median observed estimates are denoted by $\times$. The light gray dots denote individual participant estimates. The median of the posterior predicted distribution from risk-reward estimation model is denoted by the large dot and the $95 \%$ HDI of the posterior predicted distribution is denoted by the solid black line. The estimates are shallower than an inverse risk-reward relationship (dashed line) and the variability in the estimates reduces as the reward magnitudes increase. Both of these properties are consistent with the properties derived from CET.

To assess the extent to which estimated probabilities systematically deviated from the perfect inverse risk-reward relationship statistically, we fitted a mathematical model - the risk-reward estimation model - to the data. The model predicts the estimated probabilities using the risk-reward heuristic. According to the heuristic,

$$
p(x)=c / x
$$


The variable $c$ is the cost of playing (i.e., $\$ 1$ or $€ 1$ ) and $x$ is the total payoff (typically including the cost of the gamble $c$ plus the gain $g$ ). Deviations of estimated probabilities from the quantities predicted by the risk-reward heuristic are captured by the following function: ${ }^{2}$

$$
p^{\prime}(x)=\exp \left\{-\delta[-\ln p(x)]^{\gamma}\right\}
$$

The $\gamma$ parameter measures the degree to which the risk-reward relationship is flatter than a perfect inverse risk-reward relationship. As $\gamma$ decreases, the risk-reward relationship flattens. That is, the probabilities associated with low payoffs are less extreme and those associated with high payoffs are more extreme. The $\delta$ parameter measures an overall bias in the estimates. A decrease in $\delta$ results in an overall downward shift of the probabilities. ${ }^{3}$

To capture the variability in the estimates, we assume that the observed estimates are distributed according to a beta distribution, with a mean of

$$
\mu=p^{\prime}(x)
$$

The beta distribution has a second parameter $\phi$ called the dispersion parameter. ${ }^{4}$ It determines the spread of the distribution or how noisy people are in reporting their estimates. We parameterized $\phi$ to test the prediction that as payoff size decreases the

2 This function was developed by Prelec et al. (1998) to estimate the decision weights that best describe people's decisions under risk and uncertainty (Luce, 2000). The function is convenient, since the distortions CET predicts are similar to those expected in probability weighting. However, we refrain from making any claims that these distortions explain the shapes of probability weighting functions.

${ }^{3}$ Bias has been examined in the context of the IFD principle; no systematic bias was found (Parker \& Sutherland, 1986).

4 Typically the beta distribution is characterized by the two independent parameters $a$ and $b$, where $a=\mu \phi$ and $b=(1-\mu) \phi$. The variance of the beta distribution is $\sigma^{2}=\mu(1-\mu) /[\phi+1]$. Thus, the larger $\phi$ is, the lower the variance. 
variability in the judged probabilities will increase, setting

$$
\phi=\exp \left[-\left(d_{0}+d_{1} x\right)\right]
$$

The parameter $d_{0}$ captures baseline variability in the estimates. The parameter $d_{1}$ captures the degree to which the dispersion or spread of the estimates changes across payoff levels, with $d_{1}=0$ indicating no change in the dispersion across the payoff levels. When $d_{1}<0$ there is an decrease in dispersion across payoff levels and when $d_{1}>0$ there is an increase in dispersion.

We fitted the risk-reward estimation model to the data using Bayesian estimation techniques (see Supplementary Materials for details and the OSF website for code). Briefly, the estimation model provides a better fit than does a standard beta regression model that captures the probability estimates in terms of a linear function of payoffs (see Supplementary Material for a model comparison). There is a close correspondence between the posterior predicted distributions of the model and the observed estimates (Figure 6).

Table 3 summarizes the parameter estimates of the model. The posterior estimates of $\gamma$ are below 1, and imply a flatter risk-reward relationship than found in a perfect inverse risk-reward relationship (Figure 6, dashed line). ${ }^{5}$ The posterior estimates of $d_{1}$ speak to the predicted change in variability across the payoffs (i.e., heteroscedasticity) in the estimates. As the estimates are negative, they imply a credible decrease in the dispersion of the estimates as the payoff magnitude increased (Table 3). Both results - a $\gamma$ below 1 and a negative $d_{1}$-are consistent with CET. It is worth noting that the values for $\delta$ also show that the estimates were biased away from the perfect inverse relationship in a manner consistent with individuals being optimistic in their estimates. Such a pattern, according to CET, might arise if participants believe they have a competitive advantage in the environment.

\footnotetext{
${ }^{5}$ A special case of the risk-reward estimation model where $\gamma$ and $\delta$ were set to 1 provided a substantially worse fit to the data (see Supplementary Material).
} 
Table 3

Estimated Parameters for the Risk-Reward Estimation Model

\begin{tabular}{lcc}
\hline Parameter & M & $95 \%$ HDI \\
\hline Sensitivity $\gamma$ & 0.23 & {$[0.19,0.27]$} \\
Bias $\delta$ & 1.21 & {$[1.14,1.28]$} \\
Baseline variability $d_{0}$ & 0.68 & {$[0.59,0.78]$} \\
Effect of payoff magnitude on variability $d_{1}$ & -0.10 & {$[-0.18,-0.02]$} \\
\hline
\end{tabular}

Note. The values for $d_{0}$ and $d_{1}$ are in terms of standardized payoff values.

Summary. People's probability estimates have two properties that are predicted by CET. First, probability estimates derived from the expected payoffs were, relative to a perfect inverse risk-reward relationship, less extreme for low payoffs and more extreme for high payoffs. This property is predicted to arise in landscapes with heterogeneity in resource sizes or with nonideal competitors. Second, participants' estimates were increasingly variable with decreasing payoffs. This, according to CET, is a common property resulting, for example, from sparsity of resources or competitors, or from nonideal competitors. Next, we test whether people's probability estimates are sensitive to changes in the competitive ecology and to what extent changes map onto the ecological probabilities.

\section{Do people's probability estimates change with conditions in the competitive ecology?}

Introduction and methods. To examine how different ecological conditions impact probability estimates, participants were asked to seek to rent a 3-bedroom apartment in a major capital city in which the availability of housing is limited. Participants learned that there were five types of apartments, ranging from "A" apartments to "E" apartments. "A" apartments had it all: They were spacious, full of 
light, in an excellent location, and freshly renovated, and they had a balcony. Each of the lower grade apartments had successively fewer of these characteristics so that "E" apartments had none of the desirable criteria. Participants were told to assume they had a typical income that allowed them to comfortably afford a typical "C" apartment (with half the desirable characteristics); they could also stretch to afford a typical "A" apartment.

We asked $N=800$ participants in an online study to judge the probability of obtaining each of the five apartment types under five ecological conditions: standard, new market, nonideal competitors, unsaturated market, and unequal competitors (Table 4). These five conditions reflected a subset of the boundary conditions established by CET (Table 1). By plotting the estimated probabilities as a function of the apartment types, we formed a risk-reward function for each participant in each condition. This function should reveal participants' beliefs about the risk-reward structure in each condition. Table 4 lists predictions for how this function should change relative to the standard condition in the apartment scenario. Briefly, in the new market condition, the ecological system is out of equilibrium and the risk-reward structure should not be present. Therefore, the risk-reward function should be flatter relative to the standard condition. In the unsaturated market, there should be an abundance of apartments, resulting in a flatter risk-reward function and a higher dispersion of estimated probabilities for smaller resource sizes (apartments; see Table 1 and Figure 3). In the nonideal competitors condition, competitors should have difficulty identifying the quality of the apartments. The risk-reward function should therefore again be flatter and (assuming variability in the number of resources) the estimated probabilities should be more disperse for smaller resource sizes (see Table 1 and Figure 4). Finally, in the unequal competitors condition, as participants assume that they have an advantage over their competitors, estimated probabilities of obtaining a resource should increase across all resource sizes. 
Table 4

Ecological Conditions of the Apartment Study and Relevant Predictions

\begin{tabular}{|c|c|c|c|c|}
\hline Condition & Scenario & $\begin{array}{c}\text { Flattening } \\
\text { of risk-reward }\end{array}$ & Decreasing variability & $\begin{array}{c}\text { Elevated } \\
\text { risk-reward function }\end{array}$ \\
\hline Standard & $\begin{array}{l}\text { Suppose you have been looking for an apartment for two weeks, along } \\
\text { with many other apartment hunters. Everything works as usual: You } \\
\text { and other apartment hunters rely on public webpages to identify avail- } \\
\text { able apartments and attend viewings. }\end{array}$ & - & - & - \\
\hline New Market & $\begin{array}{l}\text { Suppose you have recently been employed by a company that has been } \\
\text { building a giant new headquarters a few kilometers outside of the cap- } \\
\text { ital. You will be one of } 20,000 \text { employees there. A majority of these } \\
\text { employees will seek residence in the adjoining apartment complex. } \\
\text { Different architects have been commissioned to build these apart- } \\
\text { ments. Consequently, while there should be enough apartments in } \\
\text { total for every employee, the apartments themselves will be equipped } \\
\text { very differently. So far, only concept graphics are available and no- } \\
\text { body has seen the apartments in person. }\end{array}$ & Yes & No difference & No difference \\
\hline Nonideal Competitors & $\begin{array}{l}\text { Suppose you have been looking for an apartment for two weeks. Typ- } \\
\text { ically, you and other apartment hunters rely on public webpages to } \\
\text { identify available apartments and attend viewings. However, for the } \\
\text { past two weeks the public webpages are frequently down. Thus, people } \\
\text { only receive very infrequent and unreliable updates about apartments } \\
\text { becoming available. }\end{array}$ & Yes & Yes & No difference \\
\hline Unsaturated Market & $\begin{array}{l}\text { Suppose the city has invested in a big city development project; over } \\
\text { the past years it has built 100,000 new apartments that become avail- } \\
\text { able during your search. The number of apartment hunters remains } \\
\text { the same. }\end{array}$ & Yes & Yes & No difference \\
\hline Unequal Competitors & $\begin{array}{l}\text { Suppose you have a friend who has gotten to know several of the } \\
\text { landlords in the area. Consequently, your friend is able to tell you } \\
\text { about apartments becoming available before they are made public, } \\
\text { allowing you to view them before other apartment hunters can. }\end{array}$ & No difference & No difference & Yes \\
\hline
\end{tabular}




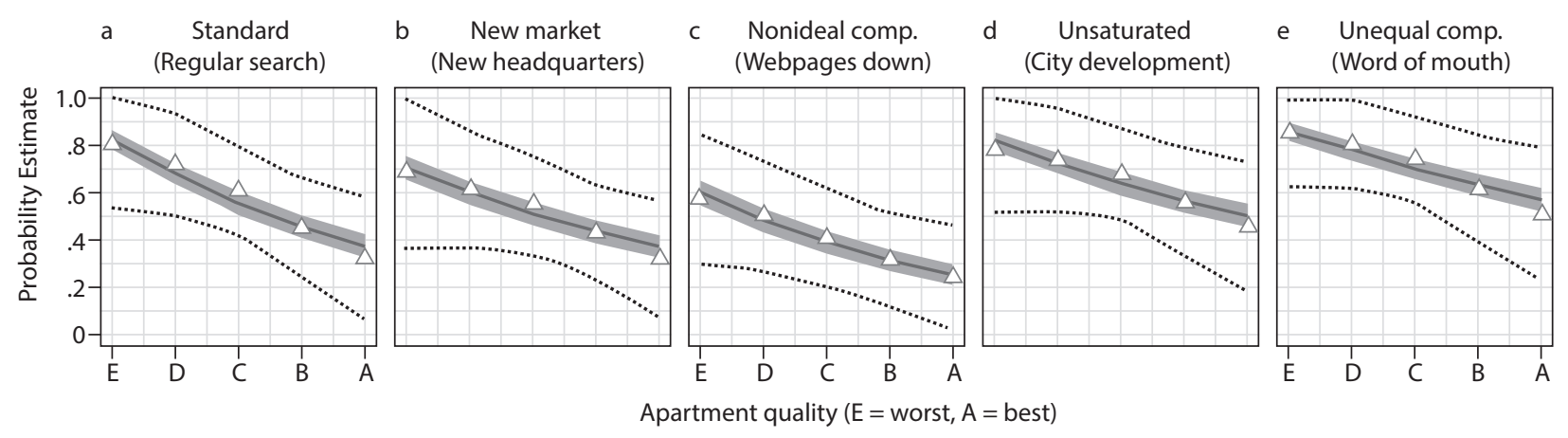

Figure 7. Probability estimates by apartment quality across five ecological conditions. The triangles denote the mean estimates, the dotted lines are the standard deviations of the estimates ( $\pm 1 \mathrm{sd})$, the solid line is the mean posterior prediction, and the gray ribbon is the $95 \%$ highest density interval of the posterior predicted distribution. Note that in the regression, apartment quality was treated as a numerical predictor ranging from 0 to 1 .

Results. All participants completed all conditions. To allow us to do both a between-subjects and a within-subjects analysis on the effect of condition, an equal number of participants completed each condition first. The order of the remaining conditions was randomized. Here we review the results from the first condition that all participants completed; all comparisons are therefore between-subject comparisons. The conclusions from the within-subject comparisons are equivalent and are reported in the Supplementary Material. Figure 7 shows the probability estimates and the resulting risk-reward functions across all five conditions. As no monetary value was assigned to apartment qualities, we did not use the risk-reward estimation model to statistically examine the effect of the environmental conditions on participants' beliefs. Instead, we used a beta regression (Smithson \& Verkuilen, 2006) in which the mean of the beta distribution was a function of the quality of the apartment as a continuous variable (ranging from 0 to 1 in equal intervals), the condition, and the interaction (via a logistic link function). We used this set of predictors to test the degree to which the slope and elevation of the risk-reward function changed. The beta regression also permitted us to simultaneously set the dispersion of the estimates as a function of the quality of the apartment as a continuous variable, the 
Table 5

Posterior Distribution Estimates of the Beta Regression Coefficients of the Mean for the First Condition of the Apartment Study

\begin{tabular}{rcr}
\hline Predictor & M & $95 \%$ HDI \\
\hline Intercept / Standard & -0.18 & {$[-0.22,-0.15]$} \\
Apartment Quality & -0.81 & {$[-0.89,-0.73]$} \\
New Market & -0.16 & {$[-0.22,-0.10]$} \\
Nonideal & -0.32 & {$[-0.39,-0.25]$} \\
Unsaturated & -0.02 & {$[-0.06,0.03]$} \\
Unequal Abilities & 0.04 & {$[-0.01,0.08]$} \\
Apartment Quality x New Market & 0.14 & {$[0.02,0.27]$} \\
Apartment Quality x Nonideal & -0.06 & {$[-0.21,0.08]$} \\
Apartment Quality x Unsaturated & 0.32 & {$[0.21,0.43]$} \\
Apartment Quality x Unequal Abilities & 0.39 & {$[0.29,0.49]$} \\
\hline
\end{tabular}

Note. In the beta regression, the mean and dispersion were parameterized to be predicted by the condition and apartment quality. This table reports the coefficients for the mean, which used a logistic link function.

condition, and the interaction (via a log link function). The regression coefficients for the mean of the beta distribution are reported in Table 5 and the coefficients for the dispersion are reported in Table 6 .

Probability estimates decreased as apartment quality increased across all ecological conditions, indicating that participants' risk-reward functions reflect the ecological risk-reward structure expected in these conditions (Figure 7). The credibly negative regression coefficient for apartment quality supports this inference (Table 5). Comparing the mean risk-reward functions across conditions shows that in every condition except the nonideal condition (Figure 7, panel c), participants adjusted their subjective probability estimates according to the ecological conditions. These adjustments were in line with CET's predictions. Specifically, the new market (panel b) and unsaturated market (panel 
Table 6

Posterior Distribution Estimates of the Beta Regression Coefficients of the Dispersion for the First Condition of the Apartment Study

\begin{tabular}{rcr}
\hline Predictor & M & $95 \%$ HDI \\
\hline Intercept / Standard & 0.60 & {$[0.45,0.75]$} \\
Apartment Quality & 0.38 & {$[0.15,0.61]$} \\
New Market & -0.34 & {$[-0.55,-0.14]$} \\
Nonideal & 0.16 & {$[-0.05,0.37]$} \\
Unsaturated & -0.21 & {$[-0.44,0.01]$} \\
Unequal Abilities & -0.06 & {$[-0.30,0.17]$} \\
Apartment Quality x New Market & 0.53 & {$[0.21,0.85]$} \\
Apartment Quality x Nonideal & 0.19 & {$[-0.14,0.52]$} \\
Apartment Quality x Unsaturated & 0.19 & {$[-0.15,0.53]$} \\
Apartment Quality x Unequal Abilities & -0.11 & {$[-0.45,0.23]$} \\
\hline
\end{tabular}

Note. In the beta regression, the mean and dispersion were

parameterized to be predicted by the condition and apartment quality. This table lists the coefficients for the dispersion, which used a log link function.

d) conditions had flatter risk-reward functions as indicated by a credible positive condition by apartment quality coefficient (see Table 5). The slope of the nonideal condition did not show a credible difference from the standard condition. One post-hoc explanation is that the prediction focuses on nonideal competitors having limited ability to accurately discriminate between apartment qualities but the scenario was worded in terms of the reliability of getting information about the availability of apartments (rather than their quality). The unequal competition condition (panel e) also showed a credibly flatter risk-reward function. We will return to this shortly.

None of the ecological conditions showed evidence in support of the prediction that variability in the estimated probabilities decreases with the increasing quality of the apartments (Table 6). 
The final prediction was that elevation of the risk-reward function would increase in the unequal-competition condition, where participants were given an unfair advantage. This was indeed the case, with participants estimating a credibly higher risk-reward function (Figure 7, panel e; Table 5). Undoubtedly, this is not the strongest test of the theory, as many other accounts would suggest that when one has a competitive advantage the estimated probability of obtaining the given resource should increase. Nevertheless, participants are sensitive to this ecological condition in a manner that is consistent with CET's prediction. We should also point out that the risk-reward function for the unequal competition condition is credibly flatter than the standard condition. This was not predicted a priori. However, a flatter function in this condition is consistent with CET's prediction that if competitors have unequal abilities throughout the environment, ignoring or collapsing across these abilities would produce a flatter risk-reward structure.

Summary. Using a competitive housing market as an ecological testing ground, we investigated how sensitive people's probability estimates are to payoffs in different ecological conditions. In all but one condition, the slope of the risk-reward function changed as a function of the ecological conditions. Moreover, the risk-reward function was elevated in the unequal competitive condition. These results are consistent with CET's predictions. However, neither the nonideal competitors condition nor the unsaturated market condition showed the predicted decrease in variability with increasing apartment size. There was, however, some evidence for this prediction in the data from the risk-reward estimation task (Table 3; Figure 6). Our results across both sets of studies suggest that people's probability estimates track the risk-reward structure and the predicted changes to it across ecological conditions, and show some limited evidence for people being sensitive to the predicted differences in variability.

\section{General Discussion}

In explaining his notion of bounded rationality, Simon (1990) wrote, 
Since we can rarely solve our problems exactly, the optimizing strategy suggested by rational analysis is seldom available. We must find techniques for solving our problems approximately, and we arrive at different solutions depending on what approximations we hit upon. Hence, to describe, predict and explain the behavior of a system of bounded rationality, we must both construct a theory of the system's processes and describe the environments to which it is adapting. (p. 7)

Simon's vision has led to extensive work on how attention, learning, memory, affective processes, and other factors shape people's judgments and decisions (for a review see Weber \& Johnson, 2009). It has also promoted an extensive cataloging of many of the choice environments in which these judgments and decisions are made (Hertwig, Pleskac, Pachur, \& the Center for Adaptive Rationality, 2019; Hertwig et al., 2013; Hogarth \& Karelaia, 2007; Marewski \& Schooler, 2011; Todd et al., 2012). One weakness of this cataloging approach is that one may end up with a different process for each discernible environmental structure, resulting in a multitude of descriptions of environment-by-process associations. In order to avoid description inflation, we suggest reframing Simon's theoretical goal as follows:

In order to describe, predict, and explain the behavior of a system of bounded rationality, one must construct theories of the system's processes as well as theories of the mechanisms behind the environmental structures to which the system is adapting.

CET is one step in this direction. It shows that the ecology of competition for scarce resources is a sufficient condition for linking risks with rewards. Since competition for limited resources is a ubiquitous condition, the risk-reward structure can be expected to be a widespread and recurrent environmental regularity. Importantly, CET also identifies how properties of the environment or properties of the competitors systematically shape the coupling of risk and reward. 


\section{An ecological theory sheds light on human behavior}

A full understanding of human behavior as part of a boundedly rational system requires an account of the environment in which behavior emerges and manifests. Indeed, it would have been difficult to predict and understand people's beliefs in the new market and unsaturated market conditions (Table 5; Figure 7) without a theory of the ecology. But CET also exposes new questions, such as: How do beliefs come to reflect ecological properties such as the risk-reward structure? Initial work has established that people are able to learn risk-reward structures as they make decisions about risky and uncertain prospects (Leuker et al., 2018). Crucially, this learning can happen incidentally, without feedback and when the person is not intending to learn anything. This may suggest that people are in some way prepared to learn this relationship - and perhaps that they even expect it.

Learning is only one of the possible mechanisms through which people adapt to ecological structures like the risk-reward structure. Social transmission may be another (Canini et al., 2014; Moussaïd et al., 2015). The fact that one finds variations of the proverb "A bird in the hand is worth two in the bush" across many cultures and languages is one indicator that the risk-reward structure may have a privileged status in human collective cognition. The German version of this proverb, for instance, has it that a sparrow in the fist is better than a pigeon on the roof, but the gist remains the same. Expressed in the probabilistic terminology of the risk-reward structure: It is better to content oneself with a smaller and safer reward than to strive for a larger but less likely one. Understanding how people pick up recurrent and stable regularities via individual, social, and cultural learning (Henrich \& McElreath, 2003; Mesoudi, 2011; Richerson \& Boyd, 2005) will be an important next step in understanding how the risk-reward structure and other environmental structures impact human behavior.

CET suggests one key ecological mechanism why payoffs and probabilities tend to be linked for people. This intercue relationship can be exploited when one cue becomes 
unavailable, as is the case when probabilities are unknown in decisions under uncertainty (Arrow, 1951; Luce \& Raiffa, 1957; Keynes, 1921; Savage, 1954). Pleskac and Hertwig (2014) suggested that this capacity is captured by Brunswik's (1952) concept of vicarious functioning: An organism can cope with cues that have become unreliable or unavailable by learning to alternate between different cues (cue substitution) through their mutual intersubstitutability. Thus, a person can gauge the probability of payoffs within a lottery by estimating the ratio of the cost of playing to the total amount of possible winnings. The direction of the inference can also be reversed: One can estimate the magnitude of unknown rewards from known risks (probabilities). Skylark \& Prabhu-Naik (2018) reported such a result. CET is as relevant for the inference from risk to rewards as it is for reversed inference from rewards to risks. For instance, the systematic change in the variability in success probabilities should affect an individual's ability to infer a reward from a given risk. In particular, small probabilities of success do not guarantee a large reward size, yet large probabilities of success will be more likely to be coupled with small resources (see Figures 3 and 5). In other words, an option that is easy to obtain is probably of low value but an option that is hard to obtain is not necessarily of high value.

\section{Ecological rationality requires an ecological theory}

The vision of ecological rationality (Todd \& Gigerenzer, 2007) highlights the fit between a heuristic - or, as Hertwig et al. (2019) highlighted, of any decision-making tool - and particular environments. However, in developing the concept of ecological rationality, the focus has been on describing the environment (Simon, 1990; Todd et al., 2012). Descriptions of environmental structures such as the risk-reward structure are not enough to understand human behavior. CET is a good illustration of why this is the case. Consider Pleskac and Hertwig's (2014) initial work on the risk-reward heuristic. Across the different environments surveyed, there was a fair amount of variation not just in the exact shape of the risk-reward structure (see Figures 3 and 4 in Pleskac \& Hertwig, 2014), but 
also in how well people's estimates mapped onto the prediction of the risk-reward heuristic. CET predicts and explains this variability in the world and in people's estimates. By drawing on the IFD principle, it identifies how key properties of the ecology (e.g., resource size, limits on the resource) as well as properties of the organisms' cognitive systems (e.g., discriminatory abilities) shape the risk-reward structure in a given environment. Without this kind of account of the environment, an understanding of the fit between decision-making tools and environments may be incomplete.

An important note of caution is in order. Not all the properties that an ecological theory deems important may shine through in people's behaviors. For instance, CET predicts the existence of a risk-reward relationship only when an organisms-environment system has reached its ecological equilibrium. When this equilibrium is not present, strictly ecologically rational actors facing uncertainty should not rely on the risk-reward structure. It is important not to postulate knowledge requirements that only omniscient actors can

meet. A person may not know that a system is not in equilibrium, and may still anchor a their thinking in the risk-reward structure, for instance via a Bayesian prior (Hoffart et al., 2018). Indeed, the results from the new market condition in our third study are consistent with this property (Figure 7, panel b). Yet the more general point is that these kinds of insights can only be established once there is a theory of the environment.

\section{A normative theory about domain-specific decision tools}

Being able to predict when the mind should and should not expect a risk-reward structure can also inform normative theories of decision making. The overarching assumption in normative decision theory is that agents maximize utility when making decisions (Keeney \& Raiffa, 1993; Savage, 1954; von Neumann \& Morgenstern, 1947). This assumption has several consequences. First, it is applicable across environments, resulting in a domain-general normative theory. Second, it is largely divorced from the properties of the agent. As Simon (1990) stated, "the economist who wishes to predict behavior studies 
the environment in which the behavior takes place, for the rational economic actor will behave in whatever way is appropriate to maximize utility in that environment" (p. 6). Thus, in some ways, expected utility theory's predictions depend entirely on the structure of the environment: All an agent has to do to act rationally is to act in accordance with the rules or axioms that permit utility maximization in that environment.

In contrast to this assumed domain generality, an adaptive approach to cognition assumes that decision makers exploit both core psychological capacities and specific ecological structures to make decisions (Anderson, 1990; Brunswik, 1943; Hertwig, Pleskac, \& Pachur, 2019; Simon, 1956; Fiedler, 2000; Kareev, 1995; Perkovic \& Orquin, 2017; Stewart et al., 2006). This suggests that the human mind draws on a repertoire of environment-specific decision strategies (Gigerenzer et al., 1999; Hertwig, Pleskac, Pachur, \& the Center for Adaptive Rationality, 2019; Payne et al., 1993), but what strategy or heuristic should be selected in a given situation? Our theory identifies a potential solution to this normative issue. First, an ecological theory can predict where and when particular decision-making tools (e.g., the risk-reward heuristic) should be applied or not. Second, it is possible to empirically test the extent to which people's behavior matches those predictions. Third, when behavior deviates from the theory's predictions, it is possible to use an ecological theory to evaluate whether the behavior was nevertheless justified and adaptive - rather than dismissing it as "irrational." Taking an ecological approach to normativity also means that the theorizing is not limited to some ideal environment. Instead, a theory of the ecology can suggest how ecologically rational actors should decide when conditions are less than ideal (Leuker et al., 2020) or when ecological anomalies occur - such as opportunities arising that are too good to be true (see Kaunitz et al., 2017; Leuker et al., 2019b). This approach is therefore highly relevant for questions about choice architecture and how to help people to improve their decision making (Thaler \& Sunstein, 2009; Johnson et al., 2012; Hertwig \& Grüne-Yanoff, 2017; Reijula et al., 2018). 


\section{Conclusion}

Nearly fifty years ago, Tversky and Kahneman (1974) argued that "people rely on a limited number of heuristic principles which reduce the complex tasks of assessing probabilities and predicting values to simpler judgmental operations. In general, these heuristics are quite useful, but sometimes they lead to severe and systematic errors" (p. 1124). The largely neglected issue in their work and the extensive work it has inspired is the question of when these heuristic principles are useful. Ecological theories like CET will help answer this question, which goes beyond simply describing and cataloging environments in which heuristics succeed or fail. As one tool in the toolkit of behavioral ecology, CET pinpoints when a risk-reward structure can reliably be expected. Inspiration for theories of the ecology will come from a wide range of sources, including physical, biological, social, institutional and cultural accounts. More ecological theories are required to better understand systems of bounded rationality and, ultimately, the coupling of mind and environment. 


\section{References}

Abrahams, M. V. (1986). Patch choice under perceptual constraints: A cause for departures from an ideal free distribution. Behavioral Ecology and Sociobiology, 19(6), 409-415. doi: 10.1007/BF00300543

Alatalo, R. V., Höglund, J., Lundberg, A., \& Sutherland, W. J. (1992). Evolution of black grouse leks: Female preferences benefit males in larger leks. Behavioral Ecology, 3(1), 53-59. doi: 10.1093/beheco/3.1.53

Anderson, J. R. (1990). The adaptive character of thought. New York, NY: Psychology Press. doi: 10.4324/9780203771730

Anderson, J. R., \& Schooler, L. J. (1991). Reflections of the environment in memory. Psychological Science, 2(6), 396-408. doi: 10.1111/j.1467-9280.1991.tb00174.x

Arrow, K. J. (1951). Alternative approaches to the theory of choice in risk-taking situations. Econometrica: Journal of the Econometric Society, 404-437. doi: $10.2307 / 1907465$

Barkow, J. H., Cosmides, L., \& Tooby, J. (1995). The adapted mind: Evolutionary psychology and the generation of culture. New York, NY: Oxford University Press.

Bloom, A. J., Chapin III, F. S., \& Mooney, H. A. (1985). Resource limitation in plants - an economic analogy. Annual Review of Ecology and Systematics, 16(1), 363-392. doi: 10.1146/annurev.es.16.110185.002051

Brunswik, E. (1943). Organismic achievement and environmental probability. Psychological Review, 50(3), 255-272. doi: 10.1037/h0060889

Brunswik, E. (1944). Distal focussing of perception: Size constancy in a representative sample of situations. Psychological Monographs, 56(254), 1-49. doi: 10.1037/h0093505 
Brunswik, E. (1952). The conceptual framework of psychology. International Encyclopaedia for Unified Science, 1, 656-670.

Canini, K. R., Griffiths, T. L., Vanpaemel, W., \& Kalish, M. L. (2014). Revealing human inductive biases for category learning by simulating cultural transmission. Psychonomic Bulletin \& Review, 21(3), 785-793. doi: 10.3758/s13423-013-0556-3

Chater, N., Oaksford, M., Hahn, U., \& Heit, E. (2010). Bayesian models of cognition. Wiley Interdisciplinary Reviews: Cognitive Science, 1(6), 811-823. doi: 10.1002/wcs.79

Chittka, L., Thomson, J. D., \& Waser, N. M. (1999). Flower constancy, insect psychology, and plant evolution. Naturwissenschaften, 86(8), 361-377. doi: 10.1007/s001140050636

Czerlinski, J., Gigerenzer, G., \& Goldstein, D. G. (1999). How good are simple heuristics? In G. Gigerenzer, P. M. Todd, \& the ABC Research Group (Eds.), Simple heuristics that make us smart (pp. 97-118). New York, NY: Oxford University Press.

Danger, M., Daufresne, T., Lucas, F., Pissard, S., \& Lacroix, G. (2008). Does Liebig's law of the minimum scale up from species to communities? Oikos, 117(11), 1741-1751. doi: 10.1111/j.1600-0706.2008.16793.x

Davies, N. B., Krebs, J. R., \& A., W. S. (2012). An introduction to behavioral ecology. 2nd ed. New York, NY: Wiley-Blackwell.

Daw, N. D., Niv, Y., \& Dayan, P. (2005). Uncertainty-based competition between prefrontal and dorsolateral striatal systems for behavioral control. Nature Neuroscience, 8(12), 1704-11. doi: 10.1038/nn1560

Dreisig, H. (1995). Ideal free distributions of nectar foraging bumblebees. Oikos, 72(2), 161-172. doi: 10.2307/3546218 
Farrior, C. E., Tilman, D., Dybzinski, R., Reich, P. B., Levin, S. A., \& Pacala, S. W. (2013). Resource limitation in a competitive context determines complex plant responses to experimental resource additions. Ecology, 94 (11), 2505-2517. doi: 10.1890/12-1548.1

Fiedler, K. (2000). Beware of samples! a cognitive-ecological sampling approach to judgment biases. Psychological Review, 107(4), 659-676. doi: 10.1037//0033-295X.107.4.695

Fox, C. R., \& Clemen, R. T. (2005). Subjective probability assessment in decision analysis: Partition dependence and bias toward the ignorance prior. Management Science, 51(9), 1417-1432. doi: 10.1287/mnsc.1050.0409

Fox, C. R., \& Levav, J. (2004). Partition-edit-count: Naive extensional reasoning in judgment of conditional probability. Journal of Experimental Psychololgy: General, 133(4), 626-42. doi: 10.1037/0096-3445.133.4.626

Fox, C. R., \& Rottenstreich, Y. (2003). Partition priming in judgment under uncertainty. Psychological Science, 14(3), 195-200. doi: 10.1111/1467-9280.02431

Fretwell, S., \& Lucas, H. (1969). On territorial behavior and other factors influencing habitat distribution in birds. Acta Biotheroretica, 19, 16-36. doi: 10.1007/BF01601953

Gershman, S. J., Horvitz, E. J., \& Tenenbaum, J. B. (2015). Computational rationality: A converging paradigm for intelligence in brains, minds, and machines. Science, 349(6245), 273-278. doi: 10.1126/science.aac6076

Gibson, J. (1979). The ecological approach to visual perception. Boston, MA: Houghton Mifflin.

Gigerenzer, G., \& Selten, R. (2002). Bounded rationality: The adaptive toolbox. Cambridge, MA: MIT Press. 
Gigerenzer, G., Todd, P. M., \& ABC Research Group. (1999). Simple heuristics that make us smart. Oxford, United Kingdom: Oxford University Press.

Gillis, D. M., Peterman, R. M., \& Tyler, A. V. (1993). Movement dynamics in a fishery: Application of the ideal free distribution to spatial allocation of effort. Canadian Journal of Fisheries and Aquatic Sciences, 50(2), 323-333. doi: 10.1139/f93-038

Goldstone, R. L., \& Ashpole, B. C. (2004). Human foraging behavior in a virtual environment. Psychonomic Bulletin \& Review, 11 (3), 508-514. doi: 10.3758/BF03196603

Goldstone, R. L., Ashpole, B. C., \& Roberts, M. E. (2005). Knowledge of resources and competitors in human foraging. Psychonomic Bulletin $\&$ Review, 12(1), 81-87. doi: $10.3758 / \mathrm{BF} 03196350$

Gorban, A. N., Pokidysheva, L. I., Smirnova, E. V., \& Tyukina, T. A. (2011). Law of the minimum paradoxes. Bulletin of Mathematical Biology, 73(9), 2013-2044. doi: $10.1007 / \mathrm{s} 11538-010-9597-1$

Griffiths, T. L., Chater, N., Kemp, C., Perfors, A., \& Tenenbaum, J. B. (2010). Probabilistic models of cognition: Exploring representations and inductive biases. Trends in Cognitive Sciences, 14, 357-364. doi: 10.1016/j.tics.2010.05.004

Harpole, W. S., Ngai, J. T., Cleland, E. E., Seabloom, E. W., Borer, E. T., Bracken, M. E., .. Smith, J. E. (2011). Nutrient co-limitation of primary producer communities. Ecology Letters, 14(9), 852-862. doi: 10.1111/j.1461-0248.2011.01651.x

Haugen, T. O., Winfield, I. J., Vøllestad, L. A., Fletcher, J. M., James, J. B., \& Stenseth, N. C. (2006). The ideal free pike: 50 years of fitness-maximizing dispersal in Windermere. Proceedings of the Royal Society B: Biological Sciences, 273(1604), 2917-2924. doi: 10.1098/rspb.2006.3659 
Henrich, J., \& McElreath, R. (2003). The evolution of cultural evolution. Evolutionary Anthropology: Issues, News, and Reviews, 12(3), 123-135. doi: 10.1002/evan.10110

Herrnstein, R. J. (1970). On the law of effect. Journal of the Experimental Analysis of Behavior, 13(2), 243-266. doi: 10.1901/jeab.1970.13-243

Herrnstein, R. J. (1974). Formal properties of the matching law. Journal of the Experimental Analysis of Behavior, 21(I), 159-164. doi: 10.1901/jeab.1974.21-159

Hertwig, R., \& Grüne-Yanoff, T. (2017). Nudging and boosting: Steering or empowering good decisions. Perspectives on Psychological Science, 12(6), 973-986. doi: $10.1177 / 1745691617702496$

Hertwig, R., Herzog, S. M., Schooler, L. J., \& Reimer, T. (2008). Fluency heuristic: A model of how the mind exploits a by-product of information retrieval. Journal of Experimental Psychology: Learning, Memory, and Cognition, 34(5), 1191. doi: $10.1037 / \mathrm{a} 0013025$

Hertwig, R., Hoffrage, U., \& ABC Research Group. (2013). Simple heuristics in a social world. New York, NY: Oxford University Press.

Hertwig, R., Pleskac, T. J., \& Pachur, T. (2019). Reckoning with uncertainty: Our research program. In Taming Uncertainty (pp. 3-28). Cambridge, MA: MIT Press. doi: $10.7551 /$ mitpress / 11114.003 .0004

Hertwig, R., Pleskac, T. J., Pachur, T., \& the Center for Adaptive Rationality. (2019). Taming uncertainty. Cambridge, MA: MIT Press.

Hoffart, J. C., Rieskamp, J., \& Dutilh, G. (2018). How environmental regularities affect people's information search in probability judgments from experience. Journal of Experimental Psychology: Learning, Memory, and Cognition. doi: 10.1037/xlm0000572 
Hogarth, R. M., \& Karelaia, N. (2007). Heuristic and linear models of judgment: Matching rules and environments. Psychological Review, 114(3), 733-758. doi: 10.1037/0033-295X.114.3.733

Holzer, H. J., Katz, L. F., \& Krueger, A. B. (1991, 8). Job queues and wages. The Quarterly Journal of Economics, 106(3), 739-768. doi: 10.2307/2937925

Houston, A. I., \& McNamara, J. M. (1988). The ideal free distribution when competitive abilities differ: An approach based on statistical mechanics. Animal Behaviour, 36(1), 166-174. doi: 10.1016/S0003-3472(88)80260-4

Johnson, E. J., Shu, S. B., Dellaert, B. G., Fox, C., Goldstein, D. G., Häubl, G., .. others (2012). Beyond nudges: Tools of a choice architecture. Marketing Letters, 23(2), 487-504. doi: 10.1007/s11002-012-9186-1

Kahneman, D., \& Frederick, S. (2002). Representativeness revisited: Attribute substitution in intuitive judgment. In T. Gilovich, D. Griffin, \& D. Kahneman (Eds.), Heuristics and biases: The psychology of intuitive judgment (pp. 49-81). Cambridge, UK: Cambridge University Press.

Kareev, Y. (1995). Through a narrow window: Working memory capacity and the detection of covariation. Cognition, 56(3), 263-269. doi: 10.1016/0010-0277(95)92814-G

Kaunitz, L., Zhong, S., \& Kreiner, J. (2017). Beating the bookies with their own numbers-and how the online sports betting market is rigged. arXiv preprint $\operatorname{arXiv:1710.02824.~}$

Keeney, R. L., \& Raiffa, H. (1993). Decisions with multiple objectives: preferences and value trade-offs. Cambridge University Press. 
Kennedy, M., \& Gray, R. D. (1993). Can ecological theory predict the distribution of foraging animals? A critical analysis of experiments on the ideal free distribution. Oikos, 68(1), 158-166. doi: 10.2307/3545322

Keynes, J. M. (1921). A treatise on probability. London, United Kingdom: Macmillan and Co.

Knight, F. H. (1921). Risk, uncertainty, and profit. New York, NY: Sentry Press.

Kozyreva, A., Pleskac, T. J., Pachur, T., \& Hertwig, R. (2019). Interpreting uncertainty: A brief history of not knowing. In (p. 343). Cambridge, MA: MIT Press. doi: $10.7551 /$ mitpress $/ 11114.003 .0026$

Krueger, A. B. (1988). The determinants of queues for federal jobs. Industrial and Labor Relations Review, 41(4), 567-581. doi: 10.3386/w2499

Kruschke, J. K. (2014). Doing Bayesian data analysis: A tutorial with R, JAGS, and Stan (2nd ed.). New York, NY: Academic Press/Elsevier.

Laplace, P. S. (1902/1814). A philosophical essay on probabilities. Wiley and Sons. (Original work published 1814)

Leuker, C., Pachur, T., Hertwig, R., \& Pleskac, T. J. (2018). Exploiting risk-reward structures in decision making under uncertainty. Cognition, 175, 186-200. doi: doi:10.1016/j.cognition.2018.02.019

Leuker, C., Pachur, T., Hertwig, R., \& Pleskac, T. J. (2019a). Do people exploit risk-reward structures to simplify information processing in risky choice? Journal of the Economic Science Association, 76-94. doi: 10.1007/s40881-019-00068-y

Leuker, C., Pachur, T., Hertwig, R., \& Pleskac, T. J. (2019b). Too good to be true? psychological responses to uncommon options in risk-reward environments. Journal of Behavioral Decision Making. doi: 10.1002/bdm.2116 
Leuker, C., Samartzidis, L., Hertwig, R., \& Pleskac, T. J. (2020). When money talks: Judging risk and coercion in high-paying clinical trials. PloS One, 15(1), e0227898. doi: 10.1371/journal.pone.0227898

Luce, R. D. (2000). Utility of gains and lossess measurement-theoretical and experimental approaches. Mahwah: Lawrence erlbaum Associates.

Luce, R. D., \& Raiffa, H. (1957). Games and decisions. New York, NY: John Wiley \& Sons, Inc.

Marewski, J. N., \& Schooler, L. J. (2011). Cognitive niches: An ecological model of strategy selection. Psychological Review, 118(3), 393-437. doi: 10.1037/a0024143

Mesoudi, A. (2011). Cultural evolution: How Darwinian theory can explain human culture and synthesize the social sciences. Chicago, IL: University of Chicago Press.

Moussaïd, M., Brighton, H., \& Gaissmaier, W. (2015). The amplification of risk in experimental diffusion chains. Proceedings of the National Academy of Sciences, 112(18), 5631-5636. doi: 10.1073/pnas.1421883112

Nicolai, C. A., Sedinger, J. S., Ward, D. H., \& Boyd, W. S. (2014). Spatial variation in life-history trade-offs results in an ideal free distribution in Black Brant Geese. Ecology, 95(5), 1323-1331. doi: 10.1890/13-0860.1

Niv, Y. (2009). Reinforcement learning in the brain. Journal of Mathematical Psychology, 53(3), 139-154. doi: 10.1016/j.jmp.2008.12.005

Parker, G. A. (1974). The reproductive behaviour and the nature of sexual selection in Scatophaga stercoraria L. (Diptera: Scatophagidae). IX. spatial distribution of fertilization rates and evolution of male search strategy within the reproductive area. Evolution, 28(1), 93-108. doi: 10.1111/j.1558-5646.1974.tb00730.x 
Parker, G. A., \& Sutherland, W. J. (1986). Ideal free distributions when individuals differ in competitive ability: Phenotype-limited ideal free models. Animal Behaviour, $34(4)$, 1222-1242. doi: 10.1016/S0003-3472(86)80182-8

Payne, J. W., Bettman, J. R., \& Johnson, E. J. (1993). The adaptive decision maker. New York, NY: Cambridge University Press.

Perkovic, S., \& Orquin, J. L. (2017). Implicit statistical learning in real-world environments leads to ecologically rational decision making. Psychological Science, 29(1), 34-44. doi: 10.1177/0956797617733831

Pleskac, T. J., \& Hertwig, R. (2014). Ecologically rational choice and the structure of the environment. Journal of Experimental Psychology: General, 143(5), 2000-2019. doi: $10.1037 /$ xge0000013

Prelec, D., et al. (1998). The probability weighting function. Econometrica, 66, 497-528.

Reijula, S., Kuorikoski, J., Ehrig, T., Katsikopoulos, K., \& Sunder, S. (2018). Nudge, boost, or design? limitations of behaviorally informed policy under social interaction. The Journal of Behavioral Economics for Policy, 2, 99-105.

Richerson, P. J., \& Boyd, R. (2005). Not by genes alone: How culture transformed human evolution. Chicago, IL: University of Chicago Press.

Rosenheim, J. A., Alon, U., \& Shinar, G. (2010). Evolutionary balancing of fitness-limiting factors. The American Naturalist, 175(6), 662-674. doi: 10.1086/652468

RStanArm version 2.9.0-4. (2016). cran.r-project.org/web/packages/rstanarm/.

Samuelson, P. A. (1965). Proof that properly anticipated prices fluctuate randomly. Industrial Management Review, 6, 41-49.

Savage, L. J. (1954). The foundations of statistics. New York, NY: John Wiley \& Sons. 
Shepard, R. N. (2001). Perceptual-cognitive universals as reflections of the world. Behavioral and brain sciences, 24(4), 581-601. doi: 10.1017/S0140525X01000012

Silver, N. (2012). The signal and the noise: why so many predictions fail-but some don't. The Penguin Press.

Simon, H. A. (1955). A behavioral model of rational choice. The Quarterly Journal of Economics, 69(1), 99-118. doi: 10.2307/1884852

Simon, H. A. (1956). Rational choice and the structure of the environment. Psychological Review, 63(2), 129-138. doi: 10.1037/h0042769

Simon, H. A. (1990). Invariants of human behavior. Annual Review of Psychology, 41, 1-19. doi: 10.1146/annurev.psych.41.1.1

Şimşek, Ö., \& Buckmann, M. (2015). Learning from small samples: An analysis of simple decision heuristics. In C. Cortes, N. D. Lawrence, D. D. Lee, M. Sugiyama, \& R. Garnett (Eds.), The 29th annual conference on neural information processing systems (pp. 3159-3167). Red Hook, NY: Curran Associates.

Skylark, W. J., \& Prabhu-Naik, S. (2018). A new test of the risk-reward heuristic. Judgment and Decision Making, 13(1), 73.

Smith, C., Reynolds, J. D., Sutherland, W. J., \& Jurajda, P. (2000, 6). Adaptive host choice and avoidance of superparasitism in the spawning decisions of bitterling (Rhodeus sericeus). Behavioral Ecology and Sociobiology, 48(1), 29-35. doi: $10.1007 / \mathrm{s} 002650000212$

Smithson, M., \& Verkuilen, J. (2006). A better lemon squeezer? maximum-likelihood regression with beta-distributed dependent variables. Psychological Methods, 11(1), 54-71. doi: 10.1037/1082-989X.11.1.54 
Sokolowski, M. B., Tonneau, F., \& Freixa i Baqué, E. (1999). The ideal free distribution in humans: An experimental test. Psychonomic Bulletin \& Review, 6(1), 157-161. doi: $10.3758 / \mathrm{bf03210824}$

Spiegelhalter, D. J., Best, N. G., Carlin, B. P., \& Van Der Linde, A. (2002). Bayesian measures of model complexity and fit. Journal of the Royal Statistical Society: Series B (Statistical Methodology), 64(4), 583-639.

Stewart, N., Chater, N., \& Brown, G. D. A. (2006). Decision by sampling. Cognitive Psychology, 53(1), 1-26. doi: 10.1016/J.Cogpsych.2005.10.003

Sutherland, W. J. (1983, 10). Aggregation and the 'ideal free' distribution. The Journal of Animal Ecology, 52(3), 821-828. doi: 10.2307/4456

Sutherland, W. J., Townsend, C. R., \& Patmore, J. M. (1988). A test of the ideal free distribution with unequal competitors. Behavioral Ecology and Sociobiology, 23(1), 51-53. doi: 10.1007/BF00303058

Thaler, R. H., \& Sunstein, C. R. (2009). Nudge: Improving decisions about health, wealth, and happiness. Penguin.

Todd, P. M., \& Gigerenzer, G. (2007). Environments that make us smart: Ecological rationality. Current Directions in Psychological Science, 16(3), 167-171. doi: 10.1111/j.1467-8721.2007.00497.x

Todd, P. M., Gigerenzer, G., \& ABC Research Group. (2012). Ecological rationality: Intelligence in the world. New York, NY: Oxford University Press.

Tregenza, T. (1995). Building on the ideal free distribution. Advances in Ecological Research, 26, 253-307. doi: 10.1016/S0065-2504(08)60067-7

Tversky, A., \& Kahneman, D. (1974). Judgment under uncertainty: Heuristics and biases. Science, 185(4157), 1124-1131. doi: 10.1126/science.185.4157.1124 
Ungemach, C., Stewart, N., \& Reimers, S. (2011). How incidental values from the environment affect decisions about money, risk, and delay. Psychological Science, 22(2), 253-260. doi: 10.1177/0956797610396225

Valladares, G., \& Lawton, J. H. (1991). Host-plant selection in the holly leaf-miner: Does mother know best? Journal of Animal Ecology, 60(1), 227-240. doi: 10.2307/5456

van der Lee, A., Gillis, D., \& Comeau, P. (2013). Comparative analysis of the spatial distribution of fishing effort contrasting ecological isodars and discrete choice models. Canadian Journal of Fisheries and Aquatic Sciences, 71(1), 141-150. doi: 10.1139/cjfas-2012-0511

von Neumann, J., \& Morgenstern, O. (1947). Theory of games and economic behavior. Princeton, NJ: Princeton University Press.

Waser, N. M. (1986). Flower constancy: Definition, cause, and measurement. The American Naturalist, 127(5), 593-603. doi: 10.1086/284507

Weber, E. U., \& Johnson, E. J. (2009). Mindful judgment and decision making. Annual Review of Psychology, 60, 53-85. doi: 10.1146/Annurev.Psych.60.110707.163633 
Appendix A

Risk-Reward and Heterogeneous Patches

Here we examine what happens to the predicted risk-reward relationship when we relax the assumption that resource sizes are constant within patches.

\section{Two resource types}

As a first step, we consider a relatively simple environment that has two resource sizes: small and large, with the constraint that $s_{\text {large }}>s_{\text {small }}=1$. We further assume that across the landscape there are two types of patches: rich and poor. A rich patch contains more large resources than small ones, $m_{\text {large }}>m_{\text {small }}$. Similarly, a poor patch contains more small resources than large ones, $m_{\text {small }}>m_{\text {large }}$.

It is useful to further specify the relationship between resource sizes in the rich and poor patches. To do so, we set the number of large resources in the rich patch and the number of small resources in the poor patch to

$$
m^{\prime}=m_{\text {rich, } \text { large }}=m_{\text {poor, } \text { small }} .
$$

We also set the number of small resources in the rich patch and the number of large resources in the poor patch to

$$
m_{\text {rich, } \text { large }}=m_{\text {poor, small }}=m^{\prime} / \mu \text {. }
$$

The parameter $\mu \geq 1$ determines the similarity of the patches across the landscapes. As $\mu \rightarrow 1$ the rich and poor patches become more and more similar to each other, but also more heterogeneous in terms of resource size. That is, both rich and poor patches grow to contain approximately $m^{\prime}$ large and small resources and as a result the within-patch heterogeneity of resources approaches between-patch heterogeneity. Analogously, as $\mu$ increases, rich and poor patches become more dissimilar to each other such that as $\mu \rightarrow \infty$ rich patches will contain only large resources and poor patches only small resources (i.e., 
patches become homogeneous with respect to resource size). Hence, as $\mu$ increases, the between-patch heterogeneity increases and the within-patch heterogeneity decreases.

Recall that the amount of resources available in a patch is a multiplicative function of the number and size of the number of resources, $r=m \times s$ (Equation 4 ). Thus, the total amount of resources $r$ available in rich patches is

$$
r_{\text {rich }}=s_{\text {large }} \times m^{\prime}+s_{\text {small }} \times \frac{m^{\prime}}{\mu} .
$$

In poor patches, the total amount of resources is

$$
r_{\text {poor }}=s_{\text {large }} \times \frac{m^{\prime}}{\mu}+s_{\text {small }} \times m^{\prime}
$$

According to the ideal free distribution (IFD) principle (Equation 3; and remembering that $s_{\text {small }}=1$ ), the number of competitors in the rich patch is

$$
n_{\text {rich }} \propto m^{\prime}\left(s_{\text {large }}+\frac{1}{\mu}\right) .
$$

The number of competitors in the poor patch is

$$
n_{\text {poor }} \propto m^{\prime}\left(\frac{s_{\text {large }}}{\mu}+1\right) .
$$

Generally, the risk-reward relationship is measured in terms of the size of the resource. To establish how the risk-reward relationship works in heterogeneous patches with different resource sizes, it is useful to find the average number of competitors per resource of a specific size. Assuming an equal number of rich and poor patches, the average number of competitors per large resource across the landscape of a total of $Y$ patches is

$$
\begin{aligned}
\bar{n}_{\text {large }} & =\frac{\sum_{y \in Y} m_{y, \text { large }} \times n_{y}}{\sum_{y \in Y} m_{y, \text { large }}} \\
& =\frac{m^{\prime} \times c \times m^{\prime}\left(s_{\text {large }}+\frac{1}{\mu}\right)+\frac{m^{\prime}}{\mu} \times c \times m^{\prime}\left(\frac{s_{\text {large }}}{\mu}+1\right)}{m^{\prime}+\frac{m^{\prime}}{\mu}} \\
& =c m^{\prime}\left(\frac{s_{\text {large }}+\mu^{2} s_{\text {large }}+2 \mu}{\mu^{2}+\mu}\right)
\end{aligned}
$$


where $c$ is a proportionality constant. Analogously, the average number of competitors per small resource is

$$
n_{\text {small }}=c m^{\prime}\left(\frac{1+\mu^{2}+2 s_{\text {large }} \mu}{\mu^{2}+\mu}\right) .
$$

Recall that the success probability $p$ of a patch is inversely proportional to the total number of competitors per resource, $p_{y} \propto m_{y} / n_{y}$ (Equation 6). Thus, from Equation A7 the success probability for a large resource is

$$
p_{\text {large }}=\frac{\mu^{2}+\mu}{m^{\prime} c\left(s_{\text {large }}+\mu^{2} s_{\text {large }}+2 \mu\right)}
$$

and for a small resource is

$$
p_{\text {small }}=\frac{\mu^{2}+\mu}{m^{\prime} c\left(1+\mu^{2}+2 s_{\text {large }} \mu\right)} .
$$

With the number of competitors and the success probability established for rich and poor patches, we can use the parameter $\mu$ to establish how the risk-reward relationship changes as the rich and poor patches become more similar and, as a result, as the heterogeneity between patches matches the heterogeneity within patches. To do so, we will examine the ratio of the probability of success for small resources relative to that of large resources,

$$
\frac{p_{\text {small }}}{p_{\text {large }}}=\frac{s_{\text {large }}+s_{\text {large }} \mu^{2}+2 \mu}{1+\mu^{2}+2 s_{\text {large }} \mu} .
$$

When poor and rich patches are equivalent and there is maximal within-path heterogeneity $(\mu=1)$, then, according to Equation A11, the ratio of success probabilities for small and large resources will be $1, p_{\text {small }} / p_{\text {large }}=1$ : When the number of large and small resources is equal in both rich and poor patches (maximal within-patch heterogeneity), success probability is on average equivalent for small and large resources and there is no risk-reward relationship.

Now consider what happens when the rich and poor patches in the landscape become maximally dissimilar and there is minimal within-patch heterogeneity in resource size (i.e., rich patches only have large resources and poor patches only have small resources). This 
occurs when $\mu \rightarrow \infty$. In this case, taking the limit of Equation A11 shows that $\lim _{\mu \rightarrow \infty} \frac{s_{\text {large }}+s_{\text {large }} \mu^{2}+2 \mu}{1+\mu^{2}+2 s_{\text {large }} \mu}=\lim _{\mu \rightarrow \infty} \frac{2 \mu s_{\text {large }}+2}{2 \mu+2 s_{\text {large }}}=s_{\text {large }}$. That is, as rich and poor patches become maximially dissimilar the ratio of success probabilities for small to large resources $\left(p_{\text {small }} / p_{\text {large }}\right)$ approaches $s_{\text {large }}$ and as a result the success probability grows to be inversely related to resource size (a risk-reward relationship).

To address what happens for intermediate levels of $\mu$ we need to establish how Equation A11 changes as a function $\mu$. To do so, we take the partial derivative of the function with respect to $\mu$. This shows that

$$
d\left(\frac{s_{\text {large }}+s_{\text {large }} \mu^{2}+2 \mu}{1+\mu^{2}+2 s_{\text {large }} \mu}\right) / d \mu \propto\left(s_{\text {large }}^{2}-1\right)\left(\mu^{2}-1\right)>0 .
$$

Therefore, for intermediate levels of $\mu$ when rich and poor patches are transitioning from maximally similar to maximally dissimilar, the ratio of success probabilties for small and large resources lies between 1 and $s_{\text {large }}$. That is, the success probability is smaller for large resources than for small resources, but the relationship is shallower than predicted when patches are maximially dissimilar (i.e., $p_{\text {small }} / p_{\text {large }}<s_{\text {large }}$ ). In particular, as rich and poor patches become more dissimilar (i.e., as $\mu$ increases), the relationship between the success probability and resource size approaches an inverse-proportional relationship and resource size becomes a more reliable predictor of the success probability.

\section{Multiple resource types}

In order to generalize to more complex situations with larger numbers of different reward sizes, we used numerical simulations. Please see the accompanying website at the Open Science Framework for the code. In the simulation, we assumed 10 resource sizes (ranging from $s=1,2, \ldots, 10$ ). There were $\sum m_{s}=1,000$ total resources with $m_{s}=100$ resources of each size class $s$. These resources were distributed among $Y=10$ patches following one of four procedures:

1. Each patch received 100 resources of one size class; different patches received resources of different sizes ("minimal within-patch heterogeneity"); 
2. Patches were divided into three classes, each receiving 100 resources as follows:

- Three "very poor" patches received resources drawn randomly from sizes 1 to 3;

- Three "intermediate" patches received resources drawn randomly from sizes 4 to 6

- Four "very rich" patches received resources drawn randomly from sizes 7 to 10 ("low within-patch heterogeneity");

3. Patches were divided into two classes, each receiving 100 resources as follows:

- Five "poor" patches received resources drawn randomly from sizes 1 to 5;

- Five "rich" patches received resources drawn randomly from sizes 6 to 10 ("high within-patch heterogeneity");

4. All patches received 100 resources drawn randomly from sizes 1 to 10 ("maximal within-patch heterogeneity").

For each patch, we calculated the total amount of resources using Equation 4. Next, $\sum_{y \in Y} n_{y}=1,000$ competitors were distributed among the patches according to the IFD principle so that the number of competitors was inversely proportional to the total amount of resources in a patch. The relative probability of success for each resource size was calculated by determining the inverse of the average number of competitors per resource of this size class across all patches,

$$
p_{s}=\frac{\sum_{y \in Y} m_{s, y}}{\sum_{y \in Y} n_{y} \cdot m_{s, y}} .
$$

Figure 2 plots the average probability of success across 100 iterations of each landscape. They confirm that the analytical results for two resource sizes generalizes to multiple resource sizes: As within-patch heterogeneity increases, the relationship between probability of success and resource size becomes increasingly shallower. Finally, when 
within-patch heterogeneity is maximal, probability of success is the same for all resource sizes (Figure 2d). 


\author{
Appendix B \\ Landscape Saturation
}

Assume a landscape in which the number of patches is large, but the number of competitors is small and resource density is low, so that resources are spread out thinly. Several patches will contain no competitors, either because these patches contain such small amounts of resources that they attract no competitors, or because there are simply not enough competitors for each patch. Do we still expect a negative relationship between reward size and probability of success in a nonsaturated landscape? We address this question with simulations. Please see the accompanying OSF website for the code.

\title{
Simulation procedure
}

We assumed a landscape with $Y=200$ patches, and resources of 10 sizes ranging from $s=1,2, \ldots 10$. The exact number of patches is of little relevance for the present purpose, as long as it is large relative to the number of competitors and/or number of resources. For simplicity, the number of resources of each size was presumed to be equal $\left(m_{s}=20\right)$. We further assumed that individual patches were homogeneous with respect to resource size, and that there were equal number of patches with resources of each size class, so that 20 patches contained resources of size $s=1$, another 20 patches contained resources of size $s=2$, and so on.

In each simulation, the resources of each size were randomly distributed among the 20 patches with that resource size according to a uniform distribution. Then competitors were distributed one by one in the landscape according to the maximal per capita consumption rate at the time. This ensured that competitors were distributed in an "ideal-free" manner. More specifically, when a new competitor entered the landscape the amount of resources $r_{y}$ was calculated for each of the 200 patches (Equation 4). Then the per capita consumption rate $u_{y}$ for each patch which this competitor would encounter at that time was calculated such that 


$$
u_{y}=\frac{r_{y}}{n_{y}+1}
$$

The new competitor then joined the patch with the highest per capita consumption rate at that time. If multiple patches had the same maximum uptake then the competitor was randomly placed in one of those patches. This procedure continued until all competitors were distributed.

At the end of each simulation, the success probability for a newly arriving focal competitor was calculated using the assumption that the success probability in a given patch is proportional to the ratio of the number of resources to the number of competitors in that patch (Equation 6). In particular, the success probability for patch $y$ was calculated as

$$
p_{y}=\frac{\frac{m_{y}}{n_{y}+1}}{\sum_{y \in Y} \frac{m_{y}}{n_{y}+1}} .
$$

Note that to make the estimated success probabilities comparable across different landscapes, we normalized the ratio of the number of resources to the number of competitors to the value of the sum of this ratio across the patches.

Since patches were homogeneous in terms of resource size, the success probability in a given patch was also the probability of success for the relevant resource size, $p_{s}$. Thus, in each simulation we obtained 20 estimates of the success probability for any given resource size.

We investigated low and high levels of competitor saturation of the landscape by distributing either $n=100$ total competitors across the landscape or $n=1,000$ competitors. These different population sizes of competitors either entered a landscape with low resource density with $m_{s}=100$ resources of each of 10 resource sizes (i.e., 1,000 resources in total) or a landscape with high resource density with $m_{s}=1000$ resources of each of 10 resource sizes (i.e., 10,000 resources in total). For each of the resulting four parameter combinations, we ran 1,000 simulations. 
Figure 3 summarizes the results of the simulations. We found that the median (and mean) success probability still decreases with resource size, but the more shallow the decrease, the less saturated the landscape is with respect to the number of competitors. Further, the probability of success is more variable the lower the saturation of the landscape with either competitors or resources, particularly for small resource sizes. 


\section{Appendix C \\ Unequal Competitors}

The ideal free distribution (IFD) principle assumes not only that competitors are ideal, but also that they are equally competitive in the landscape. That is, they have an equal ability to obtain a resource. To examine the consequences of this assumption we relax it, allowing for two classes of competitors: bullies and wimps. Bullies are twice as good at acquiring resources as are wimps. Thus, the presence of a bully impacts a wimp's resource consumption twice as strongly as the presence of another wimp does, while any wimp impacts on a bully's intake only half as strongly as another bully does.

With these constraints, based on an IFD, we set the per capita resource consumption rate $u$ in a given patch $y$ for bullies as

$$
u_{y, \text { bullies }}=\frac{r_{y}}{n_{y, \text { bullies }}+\frac{1}{2} n_{y, \text { wimps }}} .
$$

The per capita resource consumption rate $u$ in a given patch $y$ for wimps is

$$
u_{y, \text { wimps }}=\frac{r_{y}}{2 n_{y, \text { bullies }}+n_{y, \text { wimps }}} .
$$

It follows from Equations $\mathrm{C} 1$ and $\mathrm{C} 2$ that the per capita consumption rate for wimps is half that for bullies in each patch with both classes of competitors. An IFD of unequal competitors across patches is possible: If both bullies and wimps move between patches so as to maximize their own resource consumption, then the per capita resource consumption rate for bullies at equilibrium is the same in all patches, and that for wimps is also the same in all patches. However, the constant per capita resource consumption rate for wimps is only half as large as that for bullies,

$$
u_{\text {bullies }}=2 u_{\text {wimps }}=\kappa \text {. }
$$

It follows from Equations C1 and C3 that the distribution of competitors in each patch is

$$
n_{y, \text { bullies }}+\frac{1}{2} \cdot n_{y, \text { wimps }}=\frac{r_{y}}{\kappa}
$$


Recall that the amount of resources is determined by the number and size of resources, $r_{y}=m_{y} \times s_{y}$ (Equation 4). This implies

$$
n_{y, \text { bullies }}+\frac{1}{2} \cdot n_{y, \text { wimps }}=\frac{r_{y}}{\kappa}=\frac{m_{y} \times s_{y}}{\kappa} .
$$

We are interested in a bully's (or wimp's) success probability within a patch. Based on the resource to competitor assumption (Equation 6), the success probability for bullies is

$$
p_{y, \text { bullies }} \propto \frac{m_{y}}{n_{y, \text { bullies }}+\frac{1}{2} \cdot n_{y, \text { wimps }}} .
$$

Recall that each wimp only impacts half as much as another bully on a bully's resource uptake, and, thus, on a bully's probability of successfully gaining a resource within a given time period. Similarly, the success probability for wimps is

$$
p_{y, \text { wimps }} \propto \frac{m_{y}}{2 \cdot n_{y, \text { bullies }}+n_{y, \text { wimps }}}
$$

Substituting Equation C4 into Equations C6 and C7, we can determine the expected relationship between success probability and resource size at equilibrium. For bullies, this results in

$$
p_{y, \text { bullies }} \propto \frac{m_{y}}{\frac{m_{y} \times s_{y}}{\kappa}} .
$$

Because $\kappa$ is a constant this expression simplifies to

$$
p_{y, \text { bullies }} \propto \frac{1}{s_{y}}
$$

For wimps, their reduced competitiveness results in the success probability being proportional to

$$
p_{y, \text { wimps }} \propto \frac{m_{y}}{\frac{m_{y} \times s_{y}}{\frac{1}{2} \kappa}} .
$$

This expression simplifies to

$$
p_{y, \text { wimps }} \propto \frac{1}{2 s_{y}} .
$$

That is, for bullies and for wimps the success probabilities are different: In our example the success probability of bullies is always twice as high as that of wimps for all resource sizes. 
This reflects the fact that, as competitors, bullies are twice as strong as wimps. However, within each class of competitors, the success probability is still inversely proportional to resource size. Hence, just as for equal competitors, the success probability for an individual competitor of a given class can be predicted by resource size. The conclusions are similar when one considers more than two classes of competitors. 


\section{Appendix D \\ Interference Competition}

If competitors interfere with each other's ability to acquire resources (e.g., because they frighten off each other's prey or engage in antagonistic interactions), then the impact of competitors on the resource consumption rate $u$ is more than just proportional (Parker \& Sutherland, 1986),

$$
u \propto \frac{r_{y}}{n_{y}^{\theta}},
$$

where $\theta>1$ and captures the degree of interference. Since the per capita resource consumption rate at equilibrium is the same in all patches, the ideal free distribution (IFD) principle (Equation 3) changes to

$$
n_{y} \propto r_{y}{ }^{\frac{1}{\theta}}
$$

Because $r_{y}=m_{y} \times s_{y}$ (Equation 4$)$, it follows that

$$
n_{y} \propto\left(m_{y} \times s_{y}\right)^{\frac{1}{\theta}}
$$

The success probability is also impacted by interference such that it is no longer inversely proportional simply to the number of competitors (Equation 6). Instead we assume that the interference impacts the success probability to the same degree it impacts the per capita consumption rate so that

$$
p_{y} \propto \frac{m_{y}}{n_{y}^{\theta}} .
$$

Substituting Equation D2 into Equation D4 shows that

$$
p_{y} \propto \frac{m_{y}}{n_{y}^{\theta}}=\frac{m_{y}}{\left[\left(m_{y} \times s_{y}\right)^{\frac{1}{\theta}}\right]^{\theta}} .
$$

Thus, with interference among competitors the success probability is still inversely proportional to the size of the resources,

$$
p_{y} \propto \frac{1}{s_{y}} .
$$

That is, while interference of competitors changes the distribution of competitors (see Equation D2), it has no effect on the risk-reward relationship. 